\title{
VLT $K$-band spectroscopy of massive young stellar objects in (ultra-)compact HII regions ${ }^{\star}$
}

\author{
A. Bik $^{1,2}$, L. Kaper ${ }^{1}$, and L. B. F. M. Waters ${ }^{1,3}$ \\ 1 Astronomical Institute "Anton Pannekoek”, University of Amsterdam, Kruislaan 403, 1098 SJ Amsterdam, The Netherlands \\ e-mail: abik@eso.org \\ 2 European Southern Observatory, Karl-Schwarzschild Strasse 2, Garching-bei-München, 85748, Germany \\ 3 Instituut voor Sterrenkunde, Katholieke Universiteit Leuven, Celestijnenlaan 200B, 3001 Heverlee, Belgium
}

Received 22 November 2004 / Accepted 10 May 2006

\section{ABSTRACT}

\begin{abstract}
High-quality $K$-band spectra of strongly reddened point sources, deeply embedded in (ultra-)compact H II regions, have revealed a population of 20 young massive stars showing no photospheric absorption lines, but sometimes strong Br $\gamma$ emission. The Br $\gamma$ equivalent widths occupy a wide range (from about 1 to over $100 \AA$ ); the line widths of $100-200 \mathrm{~km} \mathrm{~s}^{-1}$ indicate a circumstellar rather than a nebular origin. The $K$-band spectra exhibit one or more features commonly associated with massive young stellar objects (YSOs) surrounded by circumstellar material: a very red colour $(J-K) \gtrsim 2$, CO bandhead emission, hydrogen emission lines (sometimes doubly peaked), and Fe II and/or Mg II emission lines. The large number of objects in our sample allows a more detailed definition and thorough investigation of the properties of the massive YSOs. In the $(K, J-K)$ colour-magnitude diagram (CMD) the massive YSO candidates are located in a region delimited by the OB zero-age main sequence, Be stars, Herbig Ae and Be stars, and $\mathrm{B}[\mathrm{e}]$ supergiants. The massive YSO distribution in the CMD suggests that the majority of the objects are of similar spectral type as the Herbig Be stars, but some of them are young $\mathrm{O}$ stars. The spectral properties of the observed objects do not correlate with the location in the CMD. The CO emission must come from a relatively dense $\left(\sim 10^{10} \mathrm{~cm}^{-3}\right)$ and hot $(T \sim 2000-5000 \mathrm{~K})$ region, sufficiently shielded from the intense UV radiation field of the young massive star. The hydrogen emission is produced in an ionised medium exposed to UV radiation. The best geometrical solution is a dense and neutral circumstellar disk causing the CO bandhead emission, and an ionised upper layer where the hydrogen lines are produced. We present arguments that the circumstellar disk is more likely a remnant of the accretion process than the result of rapid rotation and mass loss such as in $\mathrm{Be} / \mathrm{B}[\mathrm{e}]$ stars.
\end{abstract}

Key words. infrared: stars - stars: formation - stars: early-type - stars: circumstellar matter - stars: pre-main sequence

\section{Introduction}

The observational study of massive stars at the earliest evolutionary phases is a rapidly expanding field. Although over the past decades significant progress has been made in unravelling the formation process of low-mass stars (e.g. Shu et al. 1987), it is not well understood how massive stars form. The contraction timescale is short, so that already very early in their formation massive stars will produce a copious radiation field that may hamper or even reverse the accretion process (Wolfire \& Cassinelli 1987). This has led to the suggestion that stars more massive than $\sim 10 M_{\odot}$ cannot form through (spherical) accretion alone, but instead form by collisions of intermediate-mass stars (Bonnell et al. 1998). Alternatively, non-spherical accretion through a disk could solve the "radiation pressure problem" (e.g. Yorke \& Sonnhalter 2002). Therefore, the detection of circumstellar disks around young massive stars is regarded as an essential step in understanding the formation of the most massive stars. Observations at centimeter and millimeter wavelengths suggest that rotating circumstellar disks are present around highmass young stellar objects (e.g. Minier et al. 1998; Shepherd et al. 2001; Beltrán et al. 2004; Chini et al. 2004; Jiang et al. 2005; Patel et al. 2005).

* Based on observations collected at the European Southern Observatory at Paranal, Chile (ESO programmes 64. H-0425, 65. H0602, 68.C-0652 and 69.C-0448).
Newly born massive stars are deeply embedded inside their natal cloud, obscured from view by 10-100 mag of visual extinction. One of the first observable signatures is the radio and infrared emission produced by the ultra- (or hyper-)compact $\mathrm{H}$ II region that emerges when the contracting star becomes hot enough to ionise the surrounding material. Although radio and infrared observations provide information on the amount of ionising radiation, these diagnostics do not allow an accurate determination of the photospheric properties of the embedded young massive star(s) (for reviews, see e.g. Churchwell 1991; Hanson 1998; Garay \& Lizano 1999; Churchwell 2002).

Our strategy aims at the direct observation of the ionising star(s) of (ultra-) compact H II (UCHII) regions at near-infrared wavelengths, where the extinction is strongly reduced $\left(A_{\mathrm{K}} \simeq\right.$ $0.1 A_{\mathrm{V}}$ ), but the (reprocessed) emission by dust at longer wavelengths has not yet set in. Recent developments in near-infrared instrumentation have made this approach feasible. By first identifying the candidate ionising stars in IRAS sources with UCHII colours (cf. Kaper et al. 2006) and subsequent $K$-band spectroscopic follow-up (Bik et al. 2005), their properties can be determined.

It turns out that our sensitivity allowed us to detect about half of the UCHIIs in the near-infrared. As shown in Bik et al. (2005); Kaper et al. (2006) and Hanson et al. (2002), the UCHII regions are always located in larger, extended HII regions. Apart from the near-infrared counterparts of some of the UCHII regions, we have spectroscopically identified two other types of objects in 
those extended regions. The first type includes the photospherically detected OB stars. Their photospheric properties are determined by applying the spectral classification criteria as proposed by Hanson et al. (1996) and by measuring the shape of the line profiles. These objects cannot be distinguished from normal "naked" OB stars. This suggests that these stars have already reached the main-sequence phase (Hanson et al. 2002; Bik et al. 2003, 2005).

In this paper we focus on the spectroscopic properties of the second type of objects; 20 objects that we encountered in our survey of southern UCHIIs. Their spectroscopic and photometric properties differ from normal main-sequence $\mathrm{OB}$ stars and exhibit features commonly associated with massive young stellar objects. In our definition, a massive YSO has already contracted to stellar dimensions and likely commenced with core hydrogen burning, thereby separating a massive YSO from a high-mass protostar which would still be in its contraction phase.

The observational characteristics of massive YSOs are not well defined, but include: (i) a red continuum, likely due to hot dust; (ii) a location inside a star forming region; and (iii) an emission-line spectrum (H I, Fe II, Mg II, Na I, CO first overtone emission). The detection of emission in the first overtone bands of $\mathrm{CO}$ (at $2.3 \mu \mathrm{m}$ ) is an important signature, as it is considered to be an indication for the presence of a dense circumstellar disk. $\mathrm{CO}$ first-overtone emission has rarely been detected in any astronomical object (McGregor et al. 1988). The first detection of CO bandhead emission in a massive YSO was reported by Scoville et al. $(1979,1983)$ in the Becklin-Neugebauer (BN) object. Geballe \& Persson (1987), Carr (1989) and Chandler et al. (1993) detected CO emission in another four high-luminosity YSOs: S106, NGC 2024-IRS2, S140 and GL2789. Modelling by Chandler et al. (1995) showed that vibrational temperatures of $\sim 3500 \mathrm{~K}$ are required to produce the relative band strengths and that the size of the emitting region has to be quite small $\left(\sim 1\right.$ AU) and dense $\left(n \sim 10^{10} \mathrm{~cm}^{-3}\right)$. A circumstellar disk could provide this environment, shielding the $\mathrm{CO}$ from the hot stellar radiation field. Alternatively, the $\mathrm{CO}$ might be shock-heated due to the interaction of a stellar wind with the surrounding cloud (see also Scoville et al. 1983; Tan 2004, in the case of the BN object), or emerging from the stellar wind itself (Chandler et al. 1995). It is not clear why some YSOs show CO emission and others do not.

Hanson et al. (1997) analysed the young ( $\$ 1 \mathrm{Myr})$ stellar population of M 17 and uncovered a handful of massive YSOs. $K$-band spectroscopy revealed that the hottest $\mathrm{O}$ stars are naked, while some of the later $\mathrm{O}$ and $\mathrm{B}$ stars show clear disk signatures (near-infrared excess, $\mathrm{CO}$ emission, double-lined $\mathrm{Pa} \delta$ profiles). Similar conclusions were reached by Blum and collaborators based on their systematic study of the stellar content of obscured galactic giant H II regions (Blum et al. 1999, 2000, 2001; Figuerêdo et al. 2002; Conti \& Blum 2002; Figuerêdo et al. 2005).

In the following we present $K$-band spectra of 20 candidate massive YSOs deeply embedded in UCHIIs, obtained with ESO's Very Large Telescope and ISAAC. We study the spectral characteristics of these potentially very young massive stars, and search for signatures that may reveal information on the process of their formation. In the next section (Sect. 2) we introduce our sample and describe the observations and data reduction process. In Sect. 3 the $K$-band spectra and near-infrared photometry are presented. In Sect. 4 we discuss some well-studied objects showing similar signatures as encountered in our massive YSO sample. In Sect. 5 we put the observed properties of our massive YSO sample in perspective and provide arguments that we have detected very young massive stars of which at least some are surrounded by a circumstellar disk, most likely remnant of the accretion process. We end this paper with a summary of our conclusions (Sect. 6).

\section{Sample definition and data reduction process}

The candidate massive YSOs discussed in this paper are selected from a survey of near-infrared bright point sources deeply embedded in high-mass star forming regions (Kaper et al. 2006). Based on their position in the colour-magnitude diagram, the most luminous and reddest sources, and thus the potentially newly born massive stars are identified. A medium-resolution ( $R=10000) K$-band spectrum has been obtained with ISAAC on the ESO VLT at Paranal, Chile. This spectral resolution, as well as the obtained high signal-to-noise ratio, permits an accurate correction for telluric absorption lines and enables us to discriminate between stellar and nebular emission. Given the demand on spectral resolution, a narrow slit $\left(0.3^{\prime \prime}\right)$ is used, thus limiting the $K$-band magnitude of the targets to brighter than $K \simeq 13$.

A number of objects turn out to be late-type foreground stars, but the majority are of early (OB) spectral type (Bik et al. 2005). Many show a photospheric spectrum for which the $K$-band spectral type has been determined, resulting in a reliable assessment of their stellar parameters. Apparently, these stars, though embedded in a young H II region, have already become "normal" OB main sequence stars. Several objects, however, do not show a photospheric spectrum, but exhibit a broad $\mathrm{Br} \gamma$ emission line; these objects define the sample of the present paper (Table 1). The photometric and spectral properties of the 20 candidate massive YSOs are outlined in the next section.

Long-slit $\left(120^{\prime \prime}\right) K$-band spectra of these objects are obtained in two wavelength settings, the first setting with a central wavelength of $2.134 \mu \mathrm{m}$ including $\mathrm{Br} \gamma$ ("Br $\gamma$-setting"). The spectrum covered in this setting was used to identify the objects. To further investigate their potential massive YSO nature, for 15 objects we also obtained spectra in another setting covering the first four $\mathrm{CO}$ first-overtone bands (central wavelength $2.234 \mu \mathrm{m}$ "CO-setting"). The presence of CO first-overtone emission is an important signature of massive YSOs, and modelling these bands provides information on the geometry and nature of their circumstellar material (e.g. Bik \& Thi 2004).

The observations in the $\mathrm{Br} \gamma$-setting were carried out partly in visitor mode (March 18-20, 2000) and partly in service mode (between May 8 and July 16, 2000). The observations in the CO setting were also obtained in two runs, one visitor mode run (February 21-23, 2002) and a service mode run in the period July 7 to August 2, 2002.

The spectra are electric ghost, dark- and flat-field corrected. The wavelength calibration of the spectra taken in the Br $\gamma$ setting is performed using arc spectra with the $\mathrm{IRAF}^{1}$ task identify. For the spectra obtained in the $\mathrm{CO}$ setting the wavelength calibration is performed using telluric $\mathrm{OH}$ emission lines (Rousselot et al. 2000). The accuracy of the wavelength calibration is $\sim 3 \mathrm{~km} \mathrm{~s}^{-1}$. In order to correct for telluric emission the object was "nodded" between two positions on the slit (A and B) such that the background of position A is recorded while the source is at position $\mathrm{B}$ and is subtracted from the observation where the source is at

1 IRAF is distributed by the National Optical Astronomy Observatories which are operated by the Association of Universities for Research in Astronomy, Inc., under cooperative agreement with the National Science Foundation. 
Table 1. Sample of candidate massive YSOs. Column 1: IRAS point source catalogue number; Col. 2: Name corresponding H II region; Col. 3: Number $K$-band point source (Kaper et al. 2006); Cols. 4, 5: right ascension and declination; Cols. 6, 7: $K$ and $J-K$ (from Kaper et al. 2006); Col. 8: $A_{\mathrm{V}}$ applied to deredden the sources (see text); Cols. 9, 10: Resulting absolute $K$-band magnitude and intrinsic $(J-K)_{0}$; Col. 11: Adopted distance with in between brackets the reference: B96: Bronfman et al. (1996) with the galactic rotation model of Brand \& Blitz (1993), C87: Caswell \& Haynes (1987) C91: Chini \& Neckel (1981), C95: Carpenter et al. (1995), F02: Figuerêdo et al. (2002), K89: Koempe et al. (1989), K94: Kurtz et al. (1994), K01: Karnik et al. (2001), Kpc: Kurtz, private communication, L92: Liseau et al. (1992), S90: Simpson \& Rubin (1990), T91: Tapia (1991), W89: Wood \& Churchwell (1989b), W97: Walsh et al. (1997).

\begin{tabular}{|c|c|c|c|c|c|c|c|c|c|c|}
\hline$\overline{\overline{\text { IRAS }}}$ & $\overline{\text { H II REGION }}$ & $\mathrm{nr}$ & $\overline{\alpha e(2000)}$ & $\overline{\overline{\delta(2000)}}$ & $\overline{\bar{K}}$ & $\overline{J-K}$ & $\overline{\overline{A_{\mathrm{V}}}}$ & $\overline{\overline{K_{0}}}$ & 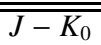 & $\overline{\bar{d}(\mathrm{kpc})}$ \\
\hline $06058+2138$ & AFGL 5180 & 227 & $06: 08: 54.8$ & $21: 38: 49.1$ & $10.7 \pm 0.03$ & $>7.0$ & 9 & -2.0 & 5.5 & 2.2 (K89) \\
\hline $06058+2138$ & AFGL 5180 & 221 & 06:08:55.1 & $21: 37: 55.5$ & $10.7 \pm 0.03$ & $3.8 \pm 0.11$ & 9 & -2.0 & 2.3 & 2.2 (K89) \\
\hline $06061+2151$ & AFGL 5182 & 676 & 06:09:07.0 & $21: 50: 31.8$ & $13.3 \pm 0.08$ & $4.2 \pm 0.50$ & 13 & 0.1 & 2.0 & 2.2 (C95) \\
\hline 06084-0611 & GGD 14 & 118 & $06: 10: 50.3$ & $-06: 11: 57.6$ & $10.8 \pm 0.03$ & $4.2 \pm 0.14$ & 17 & -1.1 & 1.3 & 1.0 (K94) \\
\hline 06084-0611 & GGD 14 & 114 & $06: 10: 50.3$ & $-06: 11: 19.4$ & $9.3 \pm 0.01$ & $4.7 \pm 0.08$ & 17 & -2.6 & 1.8 & 1.0 (K94) \\
\hline 06412-0105 & & 121 & $06: 43: 48.4$ & $-01: 08: 20.6$ & $10.2 \pm 0.02$ & $1.8 \pm 0.04$ & 11 & -5.3 & 0.0 & $7.3(\mathrm{Kpc})$ \\
\hline $07299-1651$ & DG 121 & 598 & $07: 32: 11.7$ & $-16: 58: 32.9$ & $11.2 \pm 0.03$ & $1.6 \pm 0.06$ & 10 & -0.7 & -0.1 & 1.4 (W97) \\
\hline $07299-1651$ & DG 121 & 618 & 07:32:09.8 & $-16: 58: 14.7$ & $9.4 \pm 0.01$ & $4.8 \pm 0.09$ & 14 & -2.9 & 2.4 & 1.4 (W97) \\
\hline 07299-1651 & DG 121 & 43 & $07: 32: 09.8$ & $-16: 58: 14.6$ & $9.4 \pm 0.01$ & $4.7 \pm 0.09$ & 14 & -2.9 & 2.3 & 1.4 (W97) \\
\hline $07299-1651$ & DG 121 & 314 & 07:32:09.6 & $-16: 58: 13.8$ & $11.5 \pm 0.03$ & $>6.0$ & 14 & -0.8 & 3.6 & 1.4 (W97) \\
\hline $08576-4334$ & & 292 & 08:59:21.6 & $-43: 45: 31.6$ & $9.4 \pm 0.01$ & $2.4 \pm 0.03$ & 12 & -1.2 & 0.4 & 0.7 (L92) \\
\hline $08576-4334$ & & 408 & $08: 59: 28.4$ & $-43: 46: 03.6$ & $7.3 \pm 0.01$ & $3.1 \pm 0.02$ & 12 & -3.3 & 1.1 & 0.7 (L92) \\
\hline $11097-6102$ & NGC 3576 & 1218 & $11: 11: 53.3$ & $-61: 18: 22.0$ & $8.4 \pm 0.01$ & $5.6 \pm 0.09$ & 17 & -5.8 & 2.7 & 2.8 (F02) \\
\hline $11097-6102$ & NGC 3576 & 693 & 11:11:54.6 & $-61: 18: 23.4$ & $9.2 \pm 0.01$ & $3.4 \pm 0.04$ & 17 & -4.4 & 0.5 & 2.8 (F02) \\
\hline $15411-5352$ & & 1955 & $15: 44: 59.5$ & $-54: 02: 19.2$ & $7.9 \pm 0.01$ & $4.3 \pm 0.03$ & 15 & -6.0 & 1.8 & 2.8 (B96) \\
\hline $16164-5046$ & & 3636 & $16: 20: 11.3$ & $-50: 53: 25.2$ & $9.5 \pm 0.02$ & $>8.1$ & 13 & -4.8 & 5.9 & $3.6(\mathrm{~K} 01)$ \\
\hline $16571-4029$ & RCW 116B & 1281 & $17: 00: 34.5$ & $-40: 33: 40.5$ & $9.3 \pm 0.01$ & $2.5 \pm 0.06$ & 16 & -2.7 & 1.5 & $1.0(\mathrm{C} 87)$ \\
\hline $17136-3617$ & GM 1-24 & 649 & $17: 17: 01.5$ & $-36: 20: 57.7$ & $9.5 \pm 0.01$ & $3.8 \pm 0.06$ & 21 & -4.4 & 0.3 & 2.0 (T91) \\
\hline $17258-3637$ & & 593 & $17: 29: 16.1$ & $-36: 40: 07.0$ & $9.6 \pm 0.01$ & $5.5 \pm 0.17$ & 16 & -4.0 & 2.8 & 2.3 (B96) \\
\hline $18006-2422$ & M 8 & 766 & $18: 03: 40.3$ & $-24: 22: 39.6$ & $9.4 \pm 0.01$ & $2.8 \pm 0.04$ & 7 & -2.7 & 1.7 & 1.9 (C91) \\
\hline $18507+0110$ & AFGL 2271 & 248 & $18: 53: 21.5$ & 01:14:00.1 & $10.3 \pm 0.02$ & $5.4 \pm 0.18$ & 27 & -5.6 & 0.9 & 3.7 (W89) \\
\hline
\end{tabular}

position A, and vice versa. The offset between the A and B positions was chosen such that the $\operatorname{stars}^{2}$ on the slit do not overlap. In some objects the nebular emission (in, e.g., the $\mathrm{Br} \gamma$ line) is extended so that the emission in the A image is overlapping with the emission in the B image. After subtraction this results in artifacts at the position of the nebular lines. We have inspected the 2D spectra of all sources for the presence of nebular emission and conclude that in most cases the contribution (positive or negative) of nebular emission to the stellar $(\mathrm{Br} \gamma)$ emission is negligible. However, in a few cases the nebular contribution is significant $(\sim 50 \%$ of the total flux in the case of $06412 \mathrm{nr} 121$, 17136nr649 and 18006nr766, see Fig. 2).

Telluric absorption lines were removed using stars of spectral type A, observed under identical sky conditions (for the list of standard stars, we refer to Bik et al. 2005). First, the only photospheric line $(\mathrm{Br} \gamma)$ in the spectrum of the A star needs to be divided out. It turns out that the best result is achieved when first the telluric features are removed from the $K$-band spectrum of the telluric standard using a high-resolution telluric spectrum (obtained at NSO/Kitt Peak). This spectrum is taken under very different sky conditions, so a lot of remnants are still visible in the corrected standard star spectrum, but without this "firstorder" telluric-line correction, a proper fit of Br $\gamma$ cannot be obtained. The Br $\gamma$ line is fitted by a combination of two exponential functions. The error on the resulting $\mathrm{Br} \gamma$ equivalent width (EW) of our target star is about 5\%. After the removal of the $\mathrm{Br} \gamma$ line of the A star, the telluric lines are removed by taking the ratio of the target spectrum with the obtained telluric spectrum. In the CO setting no stellar features are present in the telluric standard star spectrum. The telluric-line correction is done using the IRAF task telluric, which allows for a shift in wavelength and a scaling in line strength yielding a more accurate fit. The task

\footnotetext{
2 To increase the efficiency of our program the orientation of the slit was chosen such that at least two stars are observed simultaneously.
}

uses a cross-correlation procedure to determine the optimal shift in wavelength and the scaling factor in line strength, which can be changed interactively. The shifts are usually a few tenths of a pixel ( 1 pixel corresponds to $16 \mathrm{~km} \mathrm{~s}^{-1}$ ); also the scaling factors are modest $(\sim 10 \%)$.

\section{3. $K$-band photometric and spectral properties of massive YSO candidates}

In this section we present the $K$-band spectra of 20 objects selected from our sample of obscured point sources in high-mass star-forming regions that display a broad, spectroscopically resolved $\operatorname{Br} \gamma$ emission line. In Table 1 the objects and some of their observed properties are listed. In the remaining part of the paper we will name the objects after the first 5 digits (i.e. the right ascension) of the IRAS point source they are associated with, together with a number based on our photometry (e.g. object 227 in IRAS $06058+2138$ we refer to as $06058 \mathrm{nr} 227$, cf. Kaper et al. 2006). These objects are further characterised by a continuum slope that is on average much redder than that of the OB stars, that are also present in most of these regions, indicating that these objects possess an infrared excess. We obtained photometric data in two near-infrared bands ( $J$ and $K$ ). That is not sufficient to discriminate an IR excess from extinction in a colour-colour diagram.

In the following we first discuss the photometric properties of these objects. After that, we evaluate the spectral features detected in the Br $\gamma$ setting; subsequently, the spectra obtained in the $\mathrm{CO}$ setting are discussed.

\subsection{Position in the colour-magnitude diagram}

In Fig. 1 the objects are plotted in a $(K, J-K)$ colour-magnitude diagram. The observed $K$-band magnitude is transformed to 


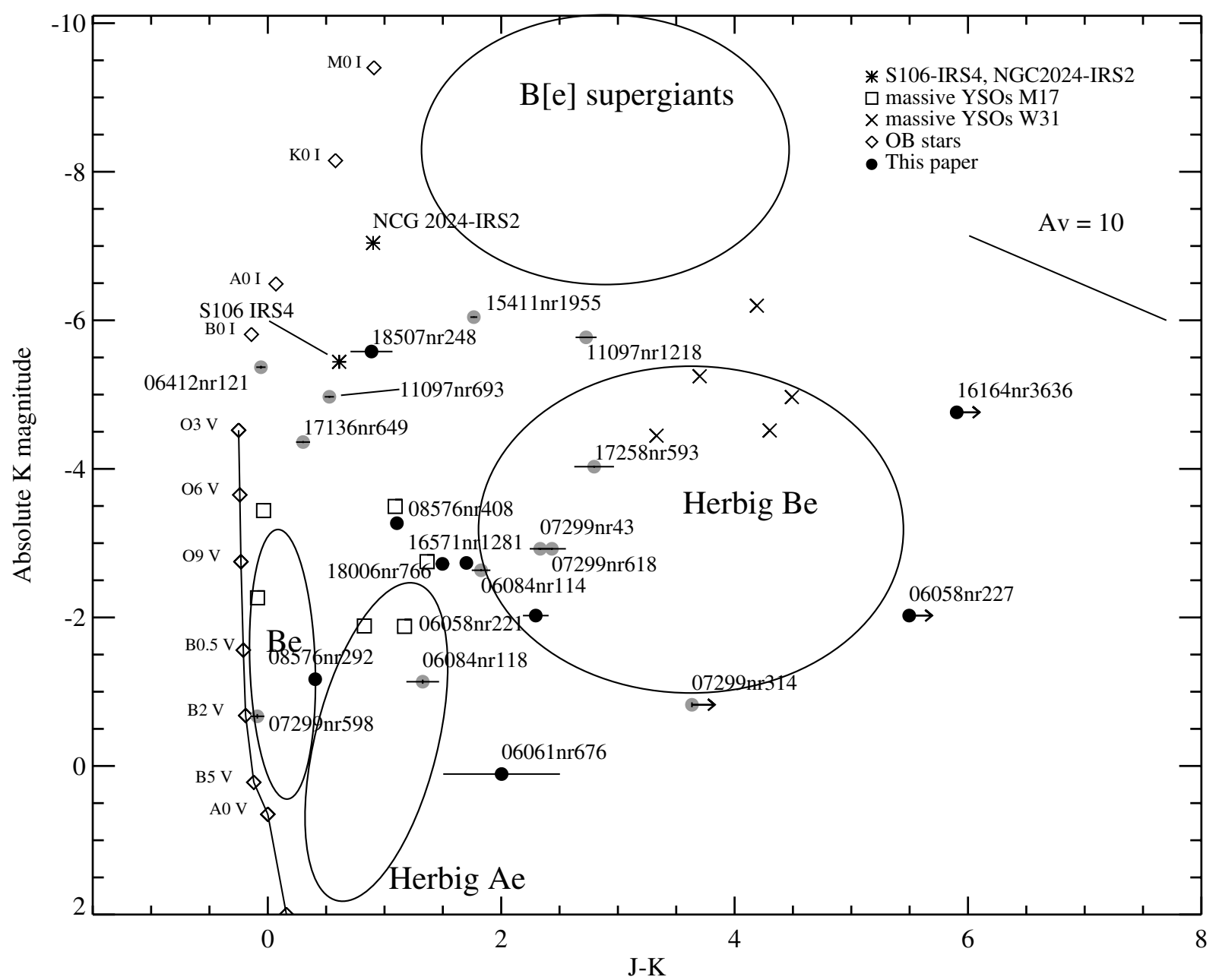

Fig. 1. Distribution of massive YSOs in the $(K, J-K)$ colour magnitude diagram. The zero-age main sequence (ZAMS) is indicated, as well as the location of some supergiants $(\diamond)$. For each object an estimate of the amount of interstellar extinction has been obtained using other members of the embedded cluster. In some cases a spectrum of a spectroscopically identified OB star (Bik et al. 2005) could be used (black circle); alternatively, an average value for the reddening is derived assuming that the other cluster members are main sequence stars (grey circle). The diagonal line in the top-right of the figure is the dereddening line representing 10 magnitudes of visual extinction. The adopted distance and the uncorrected values of $K$ and $J-K$ are listed in Table 1. The objects marked by a " $\square$ " and a " $\times$ " symbol are the extinction corrected positions of the massive YSOs found in M 17 (Hanson et al. 1997) and W31 (Blum et al. 2001), respectively. The position of NGC 2024-IRS2 (Lenorzer et al. 2004) and S106 (Felli et al. 1984) is indicated by a “ $\star$ ”. For comparison, the areas occupied by Herbig AeBe stars (Eiroa et al. 2002), B[e] stars (Voors 1999), and Be stars (Dougherty et al. 1994) are indicated.

absolute magnitude by taking into account the distance and interstellar reddening. The adopted distance is listed in Table 1 . The solid line is the location of the zero-age main sequence (ZAMS) (Hanson et al. 1997; Cox 2000).

It turns out that practically all candidate massive YSOs are members of an embedded cluster (cf. Kaper et al. 2006). The massive YSOs located in IRAS 06412-0105 and IRAS 154115352 look isolated, but those regions are associated on a larger scale with (massive) starforming regions. Our observations also do not go deep enough to exclude the presence of low-mass members of the cluster. In some cases we have been able to spectroscopically identify other early-type cluster members (Bik et al. 2005). As the intrinsic colour $(J-K)_{0}$ of all early-type main-sequence stars is $\sim-0.2$, it is then straightforward to derive the amount of interstellar reddening.

Assuming that the extinction does not vary a lot over the H II region, this method to estimate the reddening could be applied to nine objects (black circles in Fig. 1). For the other objects (grey circles) the average $(J-K)$ of the embedded cluster members was taken to measure the reddening, assuming that these stars are all on the main sequence and thus have $(J-K)_{0} \sim 0$. It may well be that the massive YSOs are more embedded compared to the already emerged OB stars, and then the derived amount of interstellar extinction is a lower limit. However, the assumption that the extinction is uniform over the $\mathrm{H}$ II region is not likely to be valid. To illustrate this with an example, the visual extinction measured in the direction of NGC 2024-IRS2 is about 3 mag higher than to NGC 2024-IRS2b, just 5" away (Lenorzer et al. 2004; Bik et al. 2003).

The objects in Fig. 1 show a wide range in $(J-K)$, from 0 to almost 6 . Also a number of objects have a very bright $K$ magnitude. If we assume that the $K$-band flux can be fully attributed to photospheric emission, the absolute $K$-band magnitude of about half of the stars would be too bright to be main sequence $\mathrm{O}$ stars and would have to be classified as supergiants. The star-forming regions containing these objects are supposed to be young and unlikely to host evolved supergiants. This is an indication that the bright $K$-band magnitude is due to a near-infrared excess.

For most UCHII regions distance determinations are not very reliable. The distances are mostly based on the radial velocities 
Table 2. Identification of the lines detected in the $K$-band spectra of candidate massive YSOs. Column 1 lists the rest wavelength, Col. 2 the corresponding element and Col. 3 the line transition.

\begin{tabular}{|c|c|c|}
\hline$\overline{\lambda(\mu \mathrm{m})}$ & Element & Transition \\
\hline 2.0893 & $\mathrm{Fe}$ II & $3 \mathrm{~d} 6.4 \mathrm{p}-3 \mathrm{~d} 6.4 \mathrm{~s}$ \\
\hline 2.1127 & $\mathrm{He} \mathrm{I}$ & $1 \mathrm{~s} .3 \mathrm{p}-1 \mathrm{~s} .4 \mathrm{~s}$ \\
\hline 2.1138 & $\mathrm{He} \mathrm{I}$ & $1 \mathrm{~s} .3 \mathrm{p}-1 \mathrm{~s} .4 \mathrm{~s}$ \\
\hline 2.1218 & $\mathrm{H}_{2}$ & $1-0 \mathrm{~S}(1)$ \\
\hline 2.1374 & $\mathrm{Mg}$ II & $5 s-5 p$ \\
\hline 2.1438 & Mg II & $5 s-5 p$ \\
\hline 2.1624 & He I & $1 \mathrm{~s} .4 \mathrm{~d}-1 \mathrm{~s} .7 \mathrm{~s}$ \\
\hline 2.1649 & $\mathrm{He} \mathrm{I}$ & $1 \mathrm{~s} .4 \mathrm{f}-1 \mathrm{~s} .7 \mathrm{~g}$ \\
\hline 2.1661 & $\mathrm{HI}(\mathrm{Br} \gamma)$ & $7-4$ \\
\hline 2.2935 & $\mathrm{CO}$ & $2-0$ \\
\hline 2.3227 & $\mathrm{CO}$ & $3-1$ \\
\hline 2.3535 & $\mathrm{CO}$ & $4-2$ \\
\hline 2.3829 & $\mathrm{CO}$ & $5-3$ \\
\hline 2.3329 & $\mathrm{HI}$ & $33-5$ \\
\hline 2.3364 & H I & $32-5$ \\
\hline 2.3402 & H I & $31-5$ \\
\hline 2.3445 & H I & $30-5$ \\
\hline 2.3492 & H I & $29-5$ \\
\hline 2.3544 & H I & $28-5$ \\
\hline 2.3603 & H I & $27-5$ \\
\hline 2.3669 & H I & $26-5$ \\
\hline 2.3743 & $\mathrm{HI}$ & $25-5$ \\
\hline 2.3828 & H I & $24-5$ \\
\hline 2.3924 & H I & $23-5$ \\
\hline
\end{tabular}

measured in molecular lines (e.g. Bronfman et al. 1996) and converted to a distance using a rotation model for the galaxy (e.g. Brand \& Blitz 1993). These distance determinations give a near and a far solution in some directions and, furthermore, the model does not account for peculiar motion. Brand \& Blitz (1993) show that in some directions the observed radial velocity spread can be up to $30-40 \mathrm{~km} \mathrm{~s}^{-1}$, which makes the distance determination uncertain. If a star of known spectral type is detected in the embedded cluster, another constraint on the distance is obtained, so that, e.g., the distance ambiguity can be resolved (cf. Bik et al. 2005).

For 5 regions a spectroscopic parallax based on optical data has been determined. IRAS 17136-3617 and IRAS 180062422 are visible in the optical, while IRAS $06058+2138$, IRAS 06061+2151 and IRAS 08576-4334 are associated with an optically visible $\mathrm{H}$ II region or $\mathrm{OB}$ association (for references, see Col. 12 of Table 1).

\subsection{Bry setting}

The objects in our sample were selected based on the presence of a broad $\mathrm{Br} \gamma$ emission line. The spectra are displayed in Fig. 2. Table 2 lists the identification of the lines detected in the spectra. The full width at half maximum $(F W H M)$ ranges from 100 to $230 \mathrm{~km} \mathrm{~s}^{-1}$ (the spectral resolution is $\sim 30 \mathrm{~km} \mathrm{~s}^{-1}$ ). The large FWHM suggests that the emission has a circumstellar (e.g. a rotating disk) and not a nebular origin. In a $\mathrm{H}$ II region of $10000 \mathrm{~K}$ the isothermal sound speed is $17 \mathrm{~km} \mathrm{~s}^{-1}$ and would remain unresolved. We note, however, that hydrogen recombination lines detected at radio wavelengths in some hyper-compact $\mathrm{H}$ II regions display comparable widths (Kurtz \& Franco 2002). In two objects (16164nr3636 and 06412nr121) He I emission lines are detected. In the case of 06412nr121 these lines are not resolved, suggesting a nebular origin. In 16164nr3636 the lines are broad and resolved like the $\mathrm{Br} \gamma$ line, and probably produced in the circumstellar environment. He I emission is only produced if the nebula is exposed to the strong UV field of a hot, early-type star (cf., e.g., Hanson et al. 2002; Martín-Hernández et al. 2003). In the majority of objects nebular, spatially extended, $\mathrm{H}_{2} 1-0 \mathrm{~S}$ (1) emission is observed. This emission is produced by molecular hydrogen that is excited by either shocks or the UV radiation field.

The strength of the $\mathrm{Br} \gamma$ emission covers a wide range (measured equivalent width $(E W)$ from 1 to over $100 \AA$, with the majority between 1 and $10 \AA$, Table 3 ). We do not find a relation between the $\mathrm{Br} \gamma$ strength and width, nor does the $\mathrm{Br} \gamma$ strength correlate with the appearance of other lines detected in this wavelength setting. One object, 06058nr221, shows a double-peaked $\mathrm{Br} \gamma$ profile, with a peak separation of $95 \mathrm{~km} \mathrm{~s}^{-1}$ (Fig. 3). Such a double-peaked profile is also observed (though in $\mathrm{Pa} \delta$ ) in massive YSOs detected in M 17 (Hanson et al. 1997). The double-peaked nature suggests the presence of a rotating disk. The Br $\gamma$ profile in several other targets displays an asymmetry. Figure 3 highlights some of the observed $\mathrm{Br} \gamma$ lines. Some objects show an additional red component, while other objects exhibit blue-shifted emission. There are no signatures indicating the presence of C IV and N III emission lines, demonstrating that these stars are not "normal" OB supergiants with a strong stellar wind causing the Br $\gamma$ emission (Hanson et al. 1996).

Several objects exhibit an Fe II emission line $(2.089 \mu \mathrm{m})$, while a few show broad Mg II emission $(2.138,2.144 \mu \mathrm{m})$. The $\mathrm{Mg}$ II emission is likely produced by the excitation through $\mathrm{Ly} \beta$ fluorescence (Bowen 1947). Like the Fe II emission, the Mg II emission lines indicate the presence of a dense and warm (several $1000 \mathrm{~K}$ ) circumstellar environment (McGregor et al. 1988).

\section{3. $C O$ setting}

\subsubsection{CO first-overtone emission}

One of the most remarkable spectral features found in these objects is the $\mathrm{CO}$ first-overtone emission in the range from 2.29-2.40 $\mu \mathrm{m}$. CO bandheads are detected in 5 sources (Fig. 2), with different shapes of the bandhead. The objects $11097 \mathrm{nr} 1218$ and 08576nr292 show a very steep bandhead, while the other 3 objects have a more shallow bandhead shape and exhibit an extended blue wing. The shape of the bandhead reflects the velocity dispersion of the CO molecules. In 11097nr1218 and 08576 nr292 the CO gas has a small velocity dispersion, which also results in a high contrast in the rotational $J$ lines. In the other objects the $\mathrm{CO}$ molecules have a much larger velocity dispersion, resulting in a reduced contrast of the $J$ lines and a broadening of the bandheads.

The observed extended blue wing of the bandhead cannot be explained by a gaussian velocity distribution. A Keplerian velocity distribution, however, can reproduce such a bandhead shape. Chandler et al. $(1993,1995)$ have observed and modelled high resolution $\mathrm{CO} 2-0$ observations of a sample of YSOs and modelled the kinematics of the CO gas. These models are based on a circumstellar accretion disk or a neutral stellar- or disk wind. Most of the objects display a CO bandhead with a blue shoulder, for which Chandler et al. (1995) obtained the best fit with a circumstellar disk. A detailed analysis and modelling of the CO first-overtone bands in our massive YSO sample support this conclusion and is presented in Bik \& Thi (2004). Similar results have been obtained by Blum et al. (2004). Contrary to the large difference in shape of the $\mathrm{CO}$ bandheads, the FWHM of $\mathrm{Br} \gamma$ is rather constant, also the CO lines with a small velocity dispersion show a broad FWHM of $\operatorname{Br} \gamma$ (Table 3). This suggests that 
Table 3. Equivalent width (EW) measurements of the emission line objects. Upper table: Cols. 2-7 give the EW for the spectral lines in the Br $\gamma$ s emission is given as positive. In Col. 8 the $F W H M$ of $\mathrm{Br} \gamma$ is given in $\mathrm{km} \mathrm{s}^{-1}$. Columns 9 and 10 show whether the CO and Pfund-lines are present i means not observed). Middle table: the EW measurements of the Pf-lines. The " $\mathrm{b}$ " means that the line could not be measured because of blending are double peaked with an average peak separation of $189 \pm 33 \mathrm{~km} \mathrm{~s}^{-1}$. Lower table: the EW measurements of the CO first-overtone bandheads. Th CO absorption lines or Pfund lines.

\begin{tabular}{|c|c|c|c|c|c|c|c|c|c|}
\hline Object & Fe II (2.09) & He I (2.1128) & He I (2.1137) & $\operatorname{Mg}$ II $(2.138)$ & Mg II (2.144) & $\operatorname{Br} \gamma(2.166)$ & $F W H M(\mathrm{Br} \gamma)$ & $\mathrm{CO}$ & $\mathrm{Pf}$ \\
\hline $06058 \mathrm{nr} 227$ & - & - & - & - & - & $1.53 \pm 0.10$ & $182 \pm 6$ & $\mathrm{n}$ & $\mathrm{n}$ \\
\hline $06058 \mathrm{nr} 221$ & - & - & - & - & - & $36.41 \pm 1.31$ & $202 \pm 1$ & $\mathrm{n}$ & $\mathrm{n}$ \\
\hline $06061 \mathrm{nr} 676$ & - & - & - & - & - & $33.22 \pm 0.34$ & $166 \pm 11$ & $\mathrm{n}$ & $\mathrm{n}$ \\
\hline $06084 \mathrm{nr} 118$ & $0.60 \pm 0.04$ & - & - & - & - & $111.1 \pm 0.12$ & $156 \pm 1$ & $\mathrm{n}$ & $\mathrm{n}$ \\
\hline $06084 \mathrm{nr} 114$ & - & - & - & - & - & $1.51 \pm 0.11$ & $212 \pm 8$ & $\mathrm{n}$ & $\mathrm{n}$ \\
\hline $06412 \mathrm{nr} 121$ & - & $0.13 \pm 0.03$ & $0.07 \pm 0.03$ & $0.78 \pm 0.18$ & $0.76 \pm 0.17$ & $12.2 \pm 0.08$ & $147 \pm 1$ & - & + \\
\hline 07299nr598 & $0.24 \pm 0.04$ & - & - & - & - & $19.6 \pm 0.13$ & $217 \pm 1$ & - & - \\
\hline 07299nr618 & $0.48 \pm 0.04$ & - & - & - & - & $4.12 \pm 0.06$ & $138 \pm 2$ & + & - \\
\hline $07299 \mathrm{nr} 43$ & $0.53 \pm 0.05$ & - & - & $0.28 \pm 0.11$ & $0.25 \pm 0.16$ & $4.87 \pm 0.08$ & $146 \pm 1$ & - & - \\
\hline $07299 \mathrm{nr} 314$ & $1.43 \pm 0.06$ & - & - & - & - & $14.9 \pm 0.11$ & $115 \pm 2$ & - & - \\
\hline 08576nr292 & - & - & - & - & - & $15.1 \pm 0.16$ & $220 \pm 2$ & + & - \\
\hline $08576 \mathrm{nr} 408$ & - & - & - & - & - & $3.88 \pm 0.09$ & $181 \pm 2$ & - & - \\
\hline $11097 \mathrm{nr} 1218$ & - & - & - & - & - & $4.83 \pm 0.08$ & $191 \pm 3$ & + & - \\
\hline $11097 \mathrm{nr} 693$ & - & - & - & - & - & $6.73 \pm 0.07$ & $121 \pm 1$ & - & - \\
\hline $15411 \mathrm{nr} 1955$ & $0.22 \pm 0.04$ & - & - & $0.47 \pm 0.10$ & $0.34 \pm 0.10$ & $6.99 \pm 0.06$ & $106 \pm 1$ & - & + \\
\hline $16164 \mathrm{nr} 3636$ & - & $2.15 \pm 0.22$ & - & $2.17 \pm 0.15$ & $1.25 \pm 0.24$ & $16.66 \pm 0.10$ & $132 \pm 1$ & + & + \\
\hline $16571 \mathrm{nr} 1281$ & - & - & - & - & - & $10.65 \pm 0.13$ & $204 \pm 1$ & - & + \\
\hline 17136nr649 & - & - & - & - & - & $6.06 \pm 0.10$ & $182 \pm 2$ & - & - \\
\hline $17258 \mathrm{nr} 593$ & $1.61 \pm 0.04$ & - & - & $0.33 \pm 0.10$ & $0.67 \pm 0.20$ & $7.98 \pm 0.06$ & $118 \pm 2$ & - & - \\
\hline $18006 \mathrm{nr} 766$ & - & $0.13 \pm 0.03$ & $0.06 \pm 0.04$ & - & - & $10.65 \pm 0.08$ & $99 \pm 1$ & + & + \\
\hline $18507 \mathrm{nr} 248$ & - & - & - & - & - & $6.41 \pm 0.07$ & $154 \pm 1$ & - & - \\
\hline Object & Pf23 & Pf24 & Pf25 & Pf26 & Pf27 & Pf28 & Pf29 & Pf30 & Pf31 \\
\hline $06412 \mathrm{nr} 121$ & $4.45 \pm 0.23$ & $3.27 \pm 0.28$ & $3.76 \pm 0.26$ & $2.61 \pm 0.27$ & $2.58 \pm 0.26$ & $2.00 \pm 0.25$ & $1.04 \pm 0.23$ & $0.94 \pm 0.21$ & $0.50 \pm 0.11$ \\
\hline 154111995 & $0.53 \pm 0.01$ & $0.41 \pm 0.01$ & $0.43 \pm 0.01$ & - & - & - & - & - & - \\
\hline $16164 \mathrm{nr} 336$ & $3.15 \pm 0.17$ & $\mathrm{~b}$ & $3.46 \pm 0.19$ & $3.20 \pm 0.20$ & $3.35 \pm 0.21$ & $\mathrm{~b}$ & $1.85 \pm 0.19$ & $2.00 \pm 0.18$ & - \\
\hline $16571 \mathrm{nr} 1281$ & $2.81 \pm 0.10$ & $2.29 \pm 0.10$ & $2.01 \pm 0.10$ & $1.33 \pm 0.10$ & $1.34 \pm 0.10$ & $1.02 \pm 0.10$ & $0.88 \pm 0.09$ & $0.53 \pm 0.08$ & $0.47 \pm 0.13$ \\
\hline $18006 \mathrm{nr} 766$ & $1.06 \pm 0.06$ & - & - & - & - & - & - & - & - \\
\hline$\overline{\text { Object }}$ & $\overline{\mathrm{CO}(2-0)}$ & $\overline{\mathrm{CO}(3-1)}$ & $\overline{\mathrm{CO}(4-2)}$ & $\overline{\mathrm{CO}(5-3)}$ & & & & & \\
\hline 07299nr618 & 4.0 & $\mathrm{~b}$ & $\mathrm{~b}$ & 1.0 & & & & & \\
\hline 08576 nr292 & 67.1 & 76.5 & 64.5 & 32.3 & & & & & \\
\hline $11097 \mathrm{nr} 1218$ & 25.0 & 28.7 & 28.7 & 19.1 & & & & & \\
\hline $16164 \mathrm{nr} 3636$ & 12.4 & $\mathrm{~b}$ & $\mathrm{~b}$ & $\mathrm{~b}$ & & & & & \\
\hline $18006 \mathrm{nr} 766$ & 17.2 & 16.2 & 12.2 & $\mathrm{~b}$ & & & & & \\
\hline
\end{tabular}




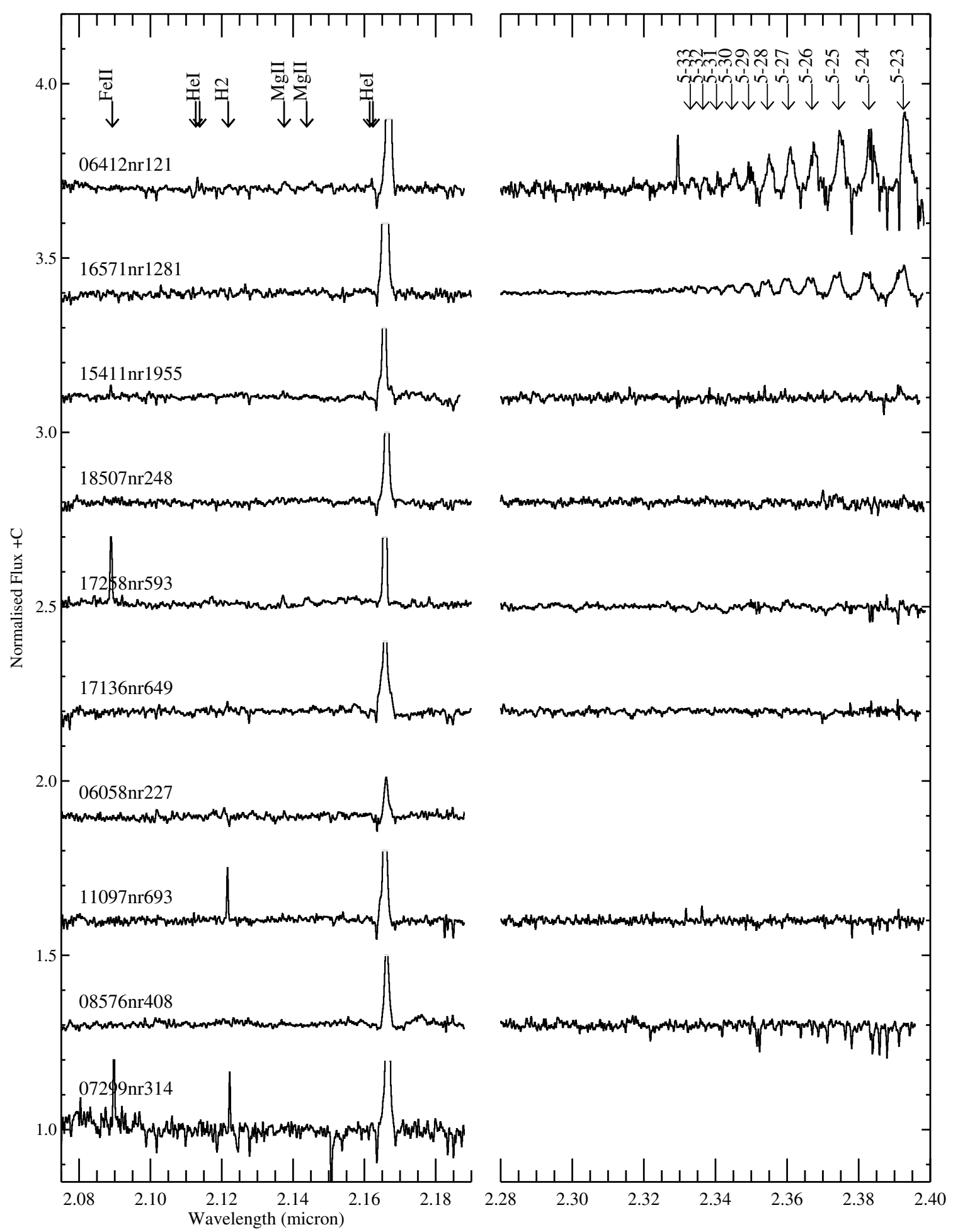

Fig. 2. $K$-band spectra of candidate massive YSOs in two wavelength settings: the Br $\gamma$ setting $(2.08-2.19 \mu \mathrm{m}$, left $)$ and the CO setting $(2.29-2.40 \mu \mathrm{m}, r i g h t)$. The spectra are normalised to the continuum. Note the gap in wavelength between the two settings. At the top of the figure the identification of the spectral lines is given. The CO setting displays the most prominent differences between the targets. Note the doublepeaked Pfund emission lines in $16571 \mathrm{nr} 1281$. The narrow absorption lines present in the CO setting of $08576 \mathrm{nr} 408$ and $17258 \mathrm{nr} 593$ are remnant telluric lines.

$\mathrm{Br} \gamma$ is not formed in the same region/geometry as the CO bandheads.

In the spectra of 16164nr3636 and 07299nr618, also CO absorption lines are detected $(2.33-2.35 \mu \mathrm{m})$. These lines are the lower $J$-lines of the $2-0$ bandhead and indicate cold $(\sim 40 \mathrm{~K})$, foreground molecular gas.

\subsubsection{Pfund lines}

In five objects hydrogen Pfund lines are detected, including two which also have the $\mathrm{CO}$ first-overtone bands in emission. In the spectrum of $16571 \mathrm{nr} 1281$ the Pf-lines are double peaked with a separation of $189 \pm 33 \mathrm{~km} \mathrm{~s}^{-1}$ suggestive of a disk origin. 

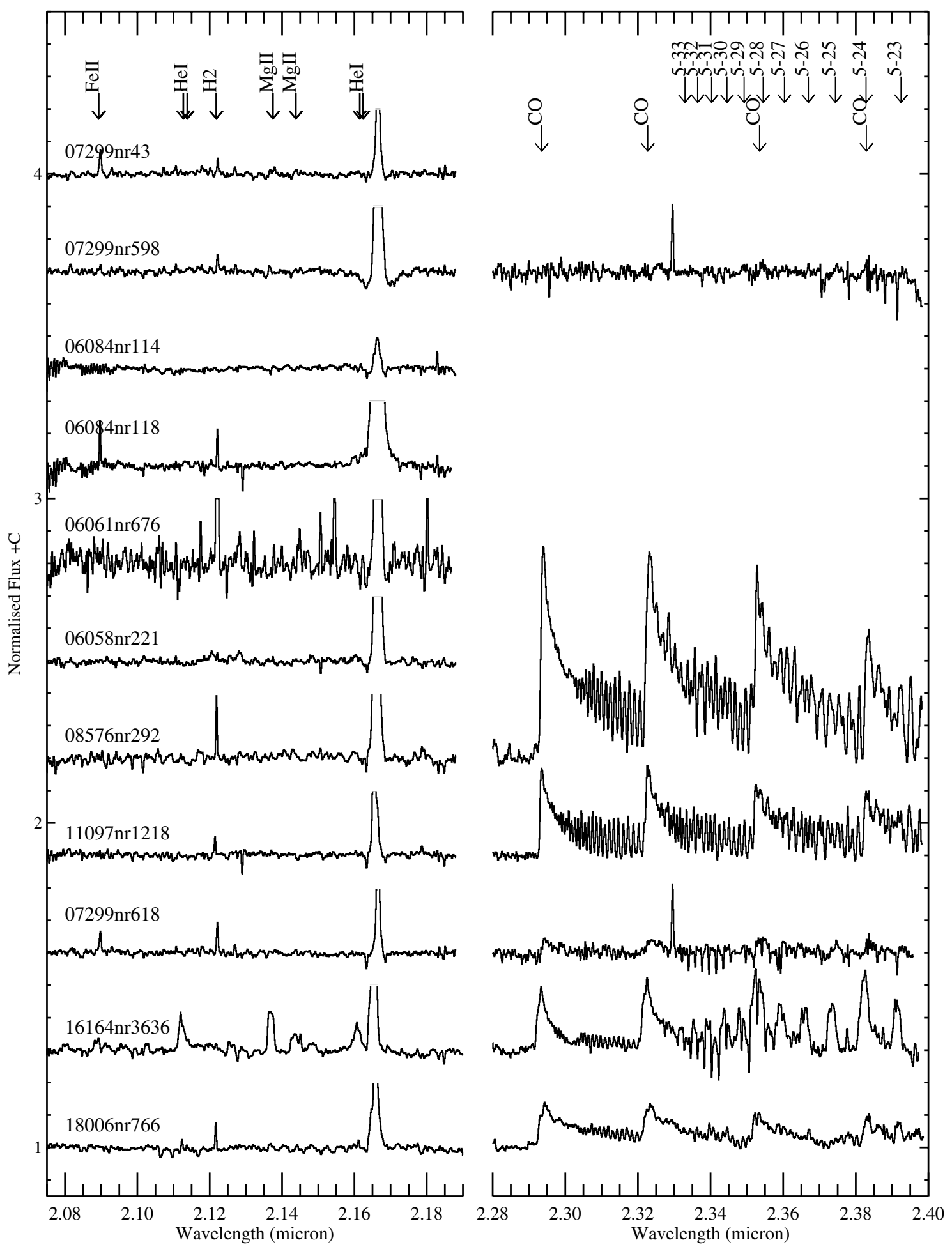

Fig. 2. Normalised $K$-band spectra of candidate massive YSOs (continued). CO first-overtone emission is detected in 5 objects with pronounced difference in line shape. $16164 \mathrm{nr} 3636$ and $18006 \mathrm{nr} 766$ show both CO and Pfund emission lines. The narrow absorption lines present in the CO setting of 07299nr598 are remnant telluric lines.

In Table 4 the $F W H M$ of Pf 25 is given in comparison with the $\mathrm{Br} \gamma$ line. It turns out that the Pf25 line is always much broader than $\mathrm{Br} \gamma$, which would be consistent with a disk origin (Sect. 5).

The Pf-line emission indicates a line forming region of high density $\left(N_{\mathrm{e}} \sim 10^{8} \mathrm{~cm}^{-3}\right)$. The flux ratio of Pf 25 and $\mathrm{Br} \gamma$ is listed in the last column of Table 4 . The ratio varies from 0.08 to 0.3 , in all cases larger than what is expected in the optically thin case B scenario $(<0.02$, Storey \& Hummer 1995). This means that when the emission of the Pf25 and $\operatorname{Br} \gamma$ are emitted by the same gas, this gas must be (partially) optically thick. However, when the two lines are produced at different locations, this ratio provides us with the ratio of the two line forming surface areas assuming that the emitting material is fully optically thick.

If the Pf-lines originate in a Keplerian disk, they are likely formed in the inner disk region, while for example Br $\gamma$ is formed in a much more extended part of the disk. In such a scenario the 

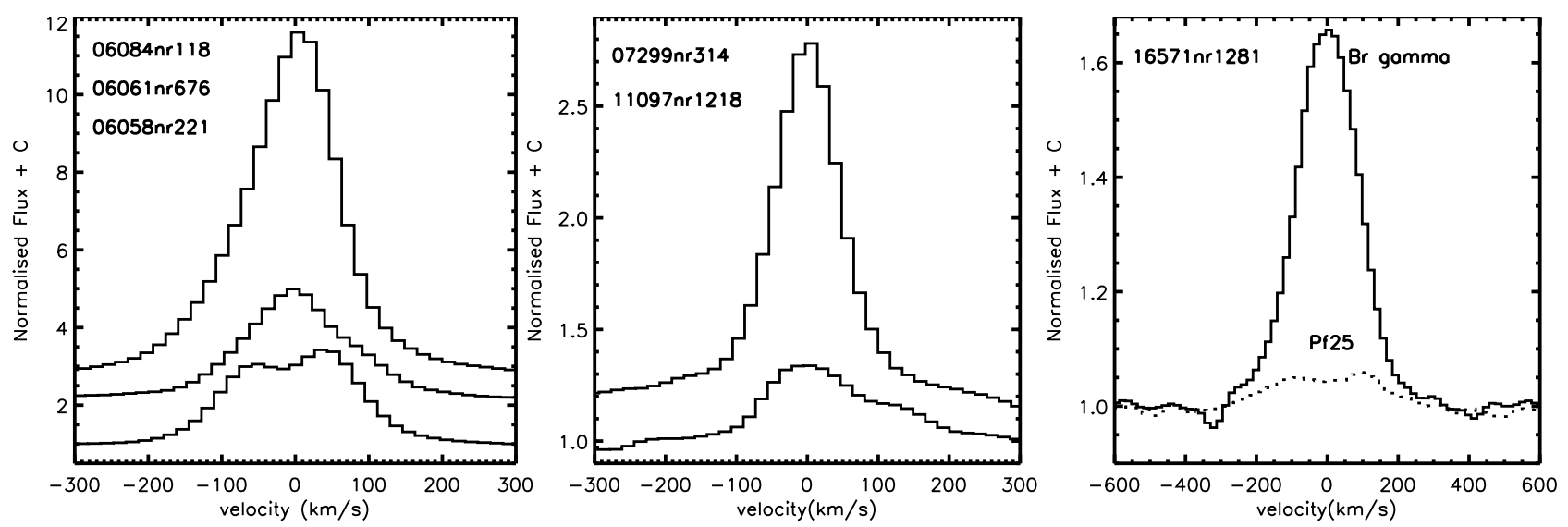

Fig. 3. Differences in Br $\gamma$ line morphology. The top spectrum in the left panel (06084nr118) shows a blue extended wing, while the bottom spectrum in the middle panel exhibits a red extended wing of the Br $\gamma$ profile. The bottom spectrum of the left panel (06058nr221) displays a double peaked Br $\gamma$ line with a peak separation of $95 \mathrm{~km} \mathrm{~s}^{-1}$, suggesting that it originates in a rotating disk. In the right panel, the Br line and Pf25 line of 16571nr1281 are plotted. By measuring the width of the two lines (Table 4), Pf25 turns out to be much broader (and double peaked) than the $\mathrm{Br} \gamma$ line. Two telluric lines at -360 and $345 \mathrm{~km} \mathrm{~s}^{-1}$ with respect to $\mathrm{Br} \gamma$ hamper an accurate determination of the Br $\gamma$ line wings.

Table 4. The FWHM of the Bry line compared with that of the hydrogen Pf25 line. The Pf 23 line is stronger than the Pf25, but this line is heavily contaminated by residuals of atmospheric lines. For the fitting of $18006 \mathrm{nr} 766$ the Pf23 line is used, because Pf25 is not detected. In the last column the flux-ratio of $\operatorname{Br} \gamma$ and $\operatorname{Pf} 25$ is listed.

\begin{tabular}{lrll}
\hline \hline \multicolumn{1}{c}{ Object } & $\begin{array}{c}F W H M \text { Br } \gamma \\
\left(\mathrm{km} \mathrm{s}^{-1}\right)\end{array}$ & $\begin{array}{c}F W H M \text { Pf25 } \\
\left(\mathrm{km} \mathrm{s}^{-1}\right)\end{array}$ & Pf25/Br $\gamma$ \\
\hline $08576 \mathrm{nr} 292$ & $220 \pm 2$ & - & - \\
$11097 \mathrm{nr} 1218$ & $191 \pm 3$ & - & - \\
$07299 \mathrm{nr} 618$ & $138 \pm 2$ & - & - \\
$16164 \mathrm{nr} 3636$ & $132 \pm 1$ & $273 \pm 8$ & 0.3 \\
$18006 \mathrm{nr} 766$ & $99 \pm 1$ & $282 \pm 13$ & 0.1 \\
$06412 \mathrm{nr} 121$ & $147 \pm 1$ & $269 \pm 7$ & 0.2 \\
$15411 \mathrm{nr} 1955$ & $106 \pm 1$ & $140 \pm 37$ & 0.08 \\
$16571 \mathrm{nr} 1281$ & $204 \pm 1$ & $328 \pm 8$ & 0.2 \\
\hline
\end{tabular}

FWHMs of the Pf-lines and $\mathrm{Br} \gamma$ are expected to be different: $\mathrm{Br} \gamma$ is predominantly formed in the more slowly rotating outer parts of the disk (larger surface area) and thus will have a smaller FWHM.

\section{Comparison to well studied objects}

In order to better understand the nature of the objects presented in this paper as well as the physical conditions under which the spectral lines are formed, the observed photometric and spectral properties of the candidate massive YSOs are compared to those of well-studied objects in the same area of the CMD.

In the following section we will first discuss some objects of which the massive YSO nature has been established based on a broad range of observations. After that we discuss the supposedly "more evolved" objects like Be and B[e] stars which show similar $K$-band spectra. In the last part of this section the observed properties of the candidate massive YSOs are discussed in detail and compared to those of the reference objects. The existing spectra of those objects are usually of worse quality and resolution than the spectra of the candidate YSOs presented in this paper. This makes a quantitative comparison difficult.

When objects have similar near-infrared spectra, this does not necessarily mean that they are in the same phase of stellar evolution; it does, however, indicate that both classes of objects provide the physical conditions under which a certain near-infrared spectrum is produced.

In Table 5 the near-infrared photometric and spectroscopic properties of the reference objects discussed in this section are summarized. Below, a short description of the special characteristics of the object or classes of objects is given.

\section{1. (Massive) Young Stellar Objects}

\section{S106-IRS4}

$\mathrm{S} 106$ is one of the prototype massive YSOs. The broad shape of the CO bandheads in the $K$-band spectrum (Table 5) is explained by emission from a narrow region in a circumstellar disk (Chandler et al. 1995). The source is further characterised by a bi-polar outflow $\left(v_{\exp }=200 \mathrm{~km} \mathrm{~s}^{-1}\right)$, with a very pronounced equatorial gap. The gap is also visible in the radio, where extinction does not play a role (Bally et al. 1983). This indicates that the gap is not caused by additional extinction, but is probably the result of a shadow produced by a circumstellar disk, which is supporting the results of the fits of the CO bandheads.

\section{BN object}

Among the YSOs, the Becklin-Neugebauer (BN) object is one of the best studied. The $\mathrm{CO}$ bandheads are rather steep. If the $\mathrm{CO}$ emission would come from a disk, the modest velocity broadening leads to the conclusion that the disk is viewed almost poleon. Scoville et al. (1983) suggest that CO emission comes from a shock-heated thin layer far from the star caused by the motion of the BN object through a cold medium.

\section{NGC 2024-IRS2}

The infrared source NGC 2024-IRS2 was discovered by Grasdalen (1974) and long thought to be the ionising source of the Flame Nebula (NGC 2024). However, Bik et al. (2003) identified NGC 2024-IRS2b as the ionising source, a late O, early B star. Using simple gas and dust models of prescribed geometry Lenorzer et al. (2004) argue that the infrared flux from the circumstellar material of NGC 2024-IRS2 is produced by a dustfree, dense gaseous disk. The density in the disk of NGC 2024IRS2 is on the order of $10^{14}-10^{15} \mathrm{~cm}^{-3}$ in the equatorial plane at the stellar surface. This is 2-3 orders of magnitude higher than in classical Be stars (Waters et al. 1991). 
Table 5. Summary of the spectral and photometric properties of the well studied objects as discussed in Sect. 4. Column 1: object name; Col. 2: spectral type; Cols. 3-6: spectral features detected in their $K$-band spectra; Cols. $7(J-K)$ value, dereddened for foreground extinction; Col. 8: absolute, dereddened $K$-band magnitude; Col. 9: references: G87: Geballe \& Persson (1987), C89: Carr (1989), C93: Chandler et al. (1993), C95: Chandler et al. (1995), vdA00: van den Ancker et al. (2000), S83: Scoville et al. (1983), L04: Lenorzer et al. (2004), vdA04: E02: Eiroa et al. (2002), van der Ancker (2004), C00: Clark \& Steele (2000), D94: Dougherty et al. (1994), M88: McGregor et al. (1988), M96: Morris et al. (1996), V99: Voors (1999). $\dagger$ : These values are for the $\mathrm{B}[\mathrm{e}]$ supergaints.

\begin{tabular}{|c|c|c|c|c|c|c|c|c|c|}
\hline $\begin{array}{c}\text { Object// } \\
\text { Object type }\end{array}$ & Sp. Type & $\mathrm{Br} \gamma$ & $\mathrm{Pf}$ & $\mathrm{HeI}$ & $\mathrm{CO}$ & MgII/FeII & $E(J-K)$ & $K$ & References \\
\hline S106 & O6-08 V & + & + & - & + & - & 0.6 & -5.4 & G87, C89, C93, C95, vdA00 \\
\hline $\mathrm{BN}$ & B $0.5 \mathrm{~V}$ & + & - & - & + & + & - & - & S83, G87, C89 \\
\hline NGC 2024-IRS2 & $\mathrm{B} 0 \mathrm{~V}$ & + & + & - & + & + & 0.9 & -7.0 & G87, C93, C95, L04 \\
\hline Herbig Ae & F - late B & + & - & - & - & - & 1.5 & $2--2$ & E02, vdA04 \\
\hline Herbig Be & $\mathrm{B}$ & + & $?$ & + & some & - & $2-6$ & $-1--5$ & $\mathrm{E} 02$, vdA04 \\
\hline $\mathrm{Be}$ & B & + & + & some & - & some & $0.0-0.6$ & $0--3$ & D94, C00 \\
\hline $\mathrm{B}[\mathrm{e}]$ & B & + & + & some & + & + & $2-6^{\dagger}$ & $-6--10^{\dagger}$ & M88,M96,V99 \\
\hline
\end{tabular}

\section{Massive YSOs in (giant) H II regions}

A near-infrared study of the young H II region M 17 by Hanson et al. (1997) has revealed a sample of massive stars possessing a near-infrared excess. The $K$-band spectral properties are identical to the objects presented here. Spectra obtained around 1 micron revealed double peaked $\operatorname{Pa} \delta$ emission, superposed on photospheric absorption lines. In the optical where the circumstellar material does not produce excess emission, the photospheric spectrum of the underlying star is visible. The spectral types derived from these spectra are early and mid-B.

\section{Herbig Ae/Be stars}

The circumstellar emission of Herbig Ae stars is produced by a passive, dusty circumstellar disk (e.g. Mannings \& Sargent 1997; Millan-Gabet et al. 2001; Eisner et al. 2003). For the Herbig Be stars the geometry of the circumstellar material is not obvious. At sub-mm wavelengths the predicted emission produced by a cold, massive circumstellar disk is not detected, suggesting that the geometry of the circumstellar material of the Herbig Be stars is different from that of the Herbig Ae stars. Natta et al. (2000) suggest that this may reflect a difference in time scales. The dispersal of the in-falling envelope by radiation pressure may trigger a rapid evolution of the circumstellar disk, and the disk will disappear much faster than in the case of Herbig Ae stars. When the Herbig Be stars become optically visible, their disks may already have disappeared. Fuente et al. (2003) found evidence for a disk around 3 Herbig Be stars, but the derived masses $\left(10^{-3}-10^{-2} M_{\odot}\right)$ are substantially lower than the mass of the disks around Herbig Ae stars $\left(10^{-2}-10^{-1} M_{\odot}\right)$.

\subsection{Main-sequence and more evolved stars}

\section{Be stars}

Be stars are rapidly rotating main sequence or (sub)giant stars that are characterised by $\mathrm{H} \alpha$ emission produced by high density circumstellar gas. Often the $\mathrm{H} \alpha$ line is double peaked. Their infrared spectra are dominated by hydrogen lines (Hony et al. 2000; Vandenbussche et al. 2002), including many from higher energy levels. Interferometric observations have confirmed the hypothesis that the circumstellar gas is distributed in a disk-like geometry (Vakili et al. 1998; Stee et al. 1998). The physical mechanism producing and maintaining these circumstellar disks is not known, but is likely related to the rapid rotation of the central star.

\section{$B[e]$ stars}

$\mathrm{B}[\mathrm{e}]$ stars show hydrogen lines in emission but also include forbidden lines in their optical spectra, mainly of [Fe II] and other low ionisation species. Contrary to Be stars, these stars exhibit a strong near-infrared and mid-infrared excess. The SED peaks between 5 and $10 \mu \mathrm{m}$, showing the signature of a hot (500-1000 K) dust component (Voors 1999). The B[e] stars comprise stars of very different nature. The classification scheme proposed by Lamers et al. (1998) includes a class of supergiants as well as a class of pre-main sequence objects. However, for many $\mathrm{B}[\mathrm{e}]$ stars the evolutionary status is unknown. The infrared excess of $\mathrm{B}[\mathrm{e}]$ supergiants is likely caused by a highly non-spherical wind which is much denser and slower at the equator than in the polar regions (Zickgraf et al. 1985). This is likely due to the rapid rotation of the star (Pelupessy et al. 2000)

In Fig. 1 the objects discussed above are plotted, together with the ZAMS and the massive YSOs presented in this paper. The Be stars are located close to the main sequence. The Herbig Be stars are much redder and bridge the gap between the Herbig Ae stars and the B[e] supergiants. The majority of the candidate massive YSOs are found near the location of the Herbig Be stars, suggesting that these objects are also of B spectral type. A small group of objects show a brighter $K$-band magnitude and also a bluer $(J-K)$ colour. They are located near the well-known massive YSO S106-IRS4. Also NGC 2024-IRS2 is found in that area of the CMD. The location of this group suggests that the central stars of these objects are of $\mathrm{O}$ spectral type, i.e. hotter and more luminous than the Herbig Be stars. For some objects, this suggestion is supported by additional arguments (see below).

\subsection{Classification of our objects}

A detailed description of the fields and stellar populations hosting the massive YSO candidates discussed in this paper is given in Kaper et al. (2006). Here we focus on the characteristics relevant for a better understanding of the physical nature of these objects. In Table 6 a summary is given of the properties of the objects. The objects will be discussed in groups based on their position in the CMD (Fig. 1).

\subsubsection{Objects with B spectral type}

The majority of the candidate massive YSOs have positions in the CMD which suggest that they are of B spectral type. A few objects hardly show any infrared excess and have colours similar to Be stars (07299nr598 and 08576nr292, Fig. 1). The spectral types of these objects (Table 6, Col. 7) are estimated by subtracting a typical $K$-band excess for Be stars $(\sim 0.6 \mathrm{mag})$. The $K$-band 
Table 6. Summary of the properties of the massive YSOs. Columns 2-4: spectral lines (-: not present, +: present, $n$ : not observed); Col. 5: is the source the counterpart of a radio UCHII?; Col. 6: spectral evidence for a circumstellar disk. (+: evidence for a disk); Col. 7: spectral type estimated based on the position in the CMD (Fig. 1); Col. 8: spectral type based on the IRAS luminosity of the IRAS source. This is an upper limit on the spectral type of the massive YSO, as usually more stars contribute to the IRAS flux. Column 9: spectral type estimate based on other methods. Column 10: properties of the surrounding cluster, taken from Kaper et al. (2006). The OB star spectra are discussed in Bik et al. (2005). References: K94: Kurtz et al. (1994, radio flux UCHII region); P03: Persi \& Tapia (2003, mid-infrared luminosity); Kpc: Kurtz, private communication (radio flux UCHII region); N84: Neckel \& Staude (1984, optical spectroscopy); C00: Clark \& Steele (2000, Bry absorption, similarity with Be stars); C87: Caswell \& Haynes (1987, single dish radio observations and assuming that a single star is responsible for the ionisation); G02: Gómez et al. (2002); B03: Barbosa et al. (2003); K01: Karnik et al. (2001).

\begin{tabular}{|c|c|c|c|c|c|c|c|c|c|}
\hline (1) & $(2)$ & (3) & (4) & (5) & (6) & (7) & (8) & (9) & (10) \\
\hline Object & $\mathrm{CO}$ & $\begin{array}{c}\mathrm{Mg} \text { II/ } \\
\text { Fe II }\end{array}$ & $\overline{\mathrm{Pf}}$ & $\overline{\mathrm{UCHII}}$ & Disk & $\begin{array}{l}\text { near-IR } \\
\text { Sp. type }\end{array}$ & $\begin{array}{l}\text { IRAS } \\
\text { Sp. type }\end{array}$ & $\begin{array}{l}\text { Other } \\
\text { Sp. type }\end{array}$ & $\begin{array}{c}\text { Cluster } \\
\text { Properties }\end{array}$ \\
\hline $06058 \mathrm{nr} 227$ & $\mathrm{n}$ & - & $\mathrm{n}$ & - & & $\mathrm{B} ?$ & B1V & & $\begin{array}{l}\text { Cluster member, B1-B2V star } \\
\text { found in same cluster. }\end{array}$ \\
\hline $06058 \mathrm{nr} 221$ & $\mathrm{n}$ & - & $\mathrm{n}$ & - & + & late $B$ & B1V & & See $06058 \mathrm{nr} 227$. \\
\hline $06061 \mathrm{nr} 676$ & $\mathrm{n}$ & - & $\mathrm{n}$ & + & & $\mathrm{B}$ ? & $\mathrm{B} 1 \mathrm{~V}$ & B2V (K94) & $\begin{array}{l}\text { Cluster member, B1-B2V star } \\
\text { found in same cluster, central } \\
\text { source UCHII region. }\end{array}$ \\
\hline $06084 \mathrm{nr} 118$ & $\mathrm{n}$ & + & $\mathrm{n}$ & + & & mid-B & $\mathrm{B} 1.5 \mathrm{~V}$ & $>\mathrm{B} 3(\mathrm{P} 03)$ & $\begin{array}{l}\text { Cluster member (GGD 14), nega- } \\
\text { tive radio slope (G02). Red mid-IR } \\
\text { SED (P03). }\end{array}$ \\
\hline $06084 \mathrm{nr} 114$ & $\mathrm{n}$ & - & $\mathrm{n}$ & - & & mid-B & $\mathrm{B} 1.5 \mathrm{~V}$ & & Cluster member. \\
\hline $06412 \mathrm{nr} 121$ & - & + & + & + & + & mid-O & $09.5 \mathrm{~V}$ & $\begin{array}{l}\text { O8V (Kpc) } \\
\text { O7V (N84) }\end{array}$ & $\begin{array}{l}\text { Nebular He I emission, ionising } \\
\text { source UCHII; brightest source. }\end{array}$ \\
\hline $07299 \mathrm{nr} 598$ & - & - & - & - & & $\mathrm{B} 2 \mathrm{~V}$ & $\mathrm{~B} 2 \mathrm{~V}$ & late B (C00) & $\begin{array}{l}\text { Likely not related to IRAS source, } \\
\text { Be star. }\end{array}$ \\
\hline 07299nr618 & + & - & - & - & + & mid-B & $\mathrm{B} 2 \mathrm{~V}$ & & $\begin{array}{l}\text { Scattered light of more embedded } \\
\text { source? }\end{array}$ \\
\hline $07299 \mathrm{nr} 43$ & + & + & - & - & & mid-B & $\mathrm{B} 2 \mathrm{~V}$ & & Blended with 07299nr43. \\
\hline $07299 \mathrm{nr} 314$ & - & + & - & - & & $\mathrm{B}$ ? & $\mathrm{B} 2 \mathrm{~V}$ & & Deeply embedded. \\
\hline 08576nr292 & + & - & - & - & + & mid-B & $\mathrm{B} 1 \mathrm{~V}$ & & $\begin{array}{l}\text { Located at edge of the cluster, } 2 \\
\text { O9V-B1V stars in center. }\end{array}$ \\
\hline $08576 n r 408$ & - & - & - & - & & early-B & B1V & & See $08576 \mathrm{nr} 292$. \\
\hline $11097 n r 1218$ & + & - & - & - & + & early-B & $\mathrm{O} 6 \mathrm{~V}$ & & $\begin{array}{l}\text { Located in cluster, not main ionis- } \\
\text { ing source, peak of radio emission } \\
\text { even more embedded. }\end{array}$ \\
\hline 11097nr693 & - & - & - & - & & late-O & $\mathrm{O} 6 \mathrm{~V}$ & & $\begin{array}{l}\text { Blue mid-IR SED (B03), located } \\
\text { close to } 11097 \mathrm{nr} 1218 .\end{array}$ \\
\hline $15411 \mathrm{nr} 1955$ & - & + & + & $\mathrm{n}$ & + & mid-O & $\mathrm{O} 8 \mathrm{~V}$ & & $\begin{array}{l}\text { Nebular He I emission, main ionis- } \\
\text { ing source H II region. No radio ob- } \\
\text { servations available. }\end{array}$ \\
\hline $16164 n r 3636$ & + & + & + & $\mathrm{n}$ & + & mid-O & $\mathrm{O} 6 \mathrm{~V}$ & $>04 \mathrm{~V}(\mathrm{C} 87)$ & $\begin{array}{l}\text { Main ionising source very com- } \\
\text { pact H II region }\left(3.5^{\prime \prime}\right) \text {; nebular He I } \\
\text { emission, embedded in massive en- } \\
\text { velope (K01). }\end{array}$ \\
\hline $16571 \mathrm{nr} 1281$ & - & - & + & - & + & early-B & $\mathrm{B} 0.5 \mathrm{~V}$ & $>09 \mathrm{~V}(\mathrm{C} 87)$ & $\begin{array}{l}\text { Cluster member, next to O8V-B1V } \\
\text { star. }\end{array}$ \\
\hline $17136 n r 649$ & - & - & - & - & & late-O & $\mathrm{O} 8 \mathrm{~V}$ & & $\begin{array}{l}\text { In center of small cluster (GM24), } \\
\text { next to UCHII region }\end{array}$ \\
\hline $17258 \mathrm{nr} 593$ & - & + & - & - & & early-B & $\mathrm{O} 8 \mathrm{~V}$ & & \\
\hline $18006 \mathrm{nr} 766$ & + & - & + & - & + & mid-B & $09.5 \mathrm{~V}$ & & $\begin{array}{l}\text { Located in M8, next to the ionising } \\
\text { source Her } 36(\mathrm{O} 7 \mathrm{~V}) \text {. }\end{array}$ \\
\hline $18507 \mathrm{nr} 248$ & - & - & - & - & & late-O & $05.5 \mathrm{~V}$ & & $\begin{array}{l}\text { Located in cluster, also } 05-06 \mathrm{~V} \\
\text { star detected }\end{array}$ \\
\hline
\end{tabular}

spectrum of 07299nr598 also has the characteristics of a Be star. Underneath the Br $\gamma$ emission line, a broad photospheric absorption component is detected. This is also seen in the late-type Be stars (Clark \& Steele 2000). The spectrum of 08576nr292, however, does not have the spectral characteristics of Be stars and shows strong $\mathrm{CO}$ bandheads.

The other objects with a larger $J-K$ are located in the same area as the Herbig Be stars. None of the stars show similarities with the Herbig Ae stars, indicating that the stars we have detected are more massive. The spectral properties, however, are less homogeneous. Some objects show CO bandheads, some Pf-lines, while other objects only show $\mathrm{Br} \gamma$ emission. This difference in spectral signature is, however, also seen in Herbig Be stars.

\subsubsection{Objects with $O$ spectral type}

A small group (5 objects) is located close to S106 in the CMD. Their luminosity suggests that the central stars are O stars. They have a relatively blue colour $(J-K \sim 0-2)$, suggesting a similar structure of the circumstellar material (Sect. 5.3). Mid-infrared data of 11097nr693 indicate a very blue SED (Barbosa et al. 2003), likely dominated by circumstellar gas instead of dust. 

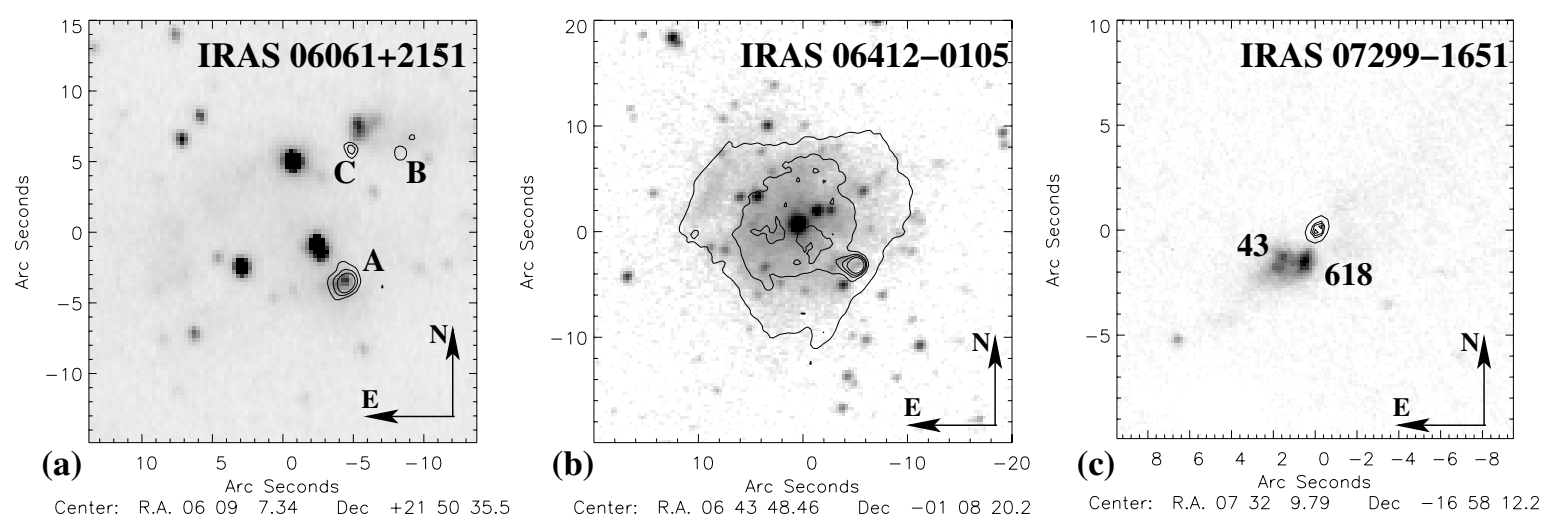

Fig. 4. $K$-band images overlayed with radio continuum data of 3 UCHII regions. Panel a): $K$-band image of IRAS 06061+2151 (Kaper et al. 2006) overlayed with a VLA $3.6 \mathrm{~cm}$ image (Kurtz et al. 1994). A (G188.796+01.030), B (G188.793+01.030) and C (G188.794+01.031) correspond to 3 UCHII regions, with UCHII region A coinciding with the 06061nr676. Panel b) $K$-band image of IRAS 06412-0105 (Kaper et al. 2006) overlayed with a VLA $1.3 \mathrm{~cm}$ image (Kurtz, priv. comm.). Panel c) VLT $K$-band acquisition image of IRAS 07299-1651, where the position of the radio source is indicated (Walsh et al. 1998). None of the three sources are coinciding with the radio source, which may be even more embedded.

The spectral properties, however, are not similar to S106, but also differ among each other. Apart from the He I emission, the spectral features do not depend strongly on the luminosity of the stars, but are much more determined by the physical conditions, like temperature and density in the circumstellar material. The main effect which seems to depend on the luminosity is the relatively blue colour of the more massive objects.

The object which resembles the spectroscopic properties of S106 most is 16164nr3636. The presence of cold CO absorption lines and the position in the CMD suggest that this object is deeply embedded. The extinction of the neighbouring OB stars used to deredden $16164 \mathrm{nr} 3636$ is obviously much less than the real extinction in this line of sight. The CO-bandheads in this object posses a blue wing which is naturally explained by a Keplerian rotating disk (Bik \& Thi 2004).

\subsubsection{Objects showing strong $\mathrm{Br} \gamma$ emission}

In the majority of the candidate massive YSOs in our sample the $\mathrm{Br} \gamma \mathrm{EW}$ is around $10 \AA$ or less. In three objects, however, the EW is significantly higher: $06058 \mathrm{nr} 221,06061 \mathrm{nr} 676$, 06084nr118 (30-100 A, Table 3). These objects are all located in the lower part of the CMD (Fig.1). They are of equal luminosity as the Herbig Ae stars, but significantly redder $(J-K \sim 2)$.

Mid-infrared observations show that $06084 \mathrm{nr} 118$ has a very red mid-infrared SED (Persi \& Tapia 2003), suggesting that the source is still heavily embedded. The IR-luminosity they derive is $350 L_{\odot}$, which corresponds to an embedded star with a spectral type later than B3. The radio spectral energy distribution has a negative slope, is variable and has a non-thermal origin. Gómez et al. (2002) suggest that the radio emission is originating in an active magnetosphere, like observed towards $\mathrm{T}$ Tauri stars. The derived IR-luminosity as well as the position in our CMD suggest that this object is much more luminous than a T Tauri star. It has a $K$-band magnitude similar to Herbig-Ae stars.

The $\mathrm{Br} \gamma$ line of $06058 \mathrm{nr} 221$ is double peaked with a separation of $95 \mathrm{~km} \mathrm{~s}^{-1}$, suggesting an origin in a rotating disk. The disk hypothesis has not been confirmed by CO or Pfund lines, as no observations were performed in this setting. 06061nr676 is one of the few objects being the near-infrared counterpart to a radio UCHII region (see Sect. 4.4), which might be contributing to the $\mathrm{Br} \gamma$ emission.

\subsection{Ionising sources of UCHII regions}

Kaper et al. (2006) find a near-infrared counterpart of an UCHII radio source in $44 \%$ of the fields containing such a source. This is in agreement with the results of Hanson et al. (2002), who show that only half of the radio UCHII regions have a near infrared counterpart.

The UCHII regions without a near-infrared counterpart are apparently still so heavily embedded that they are not detectable at near-infrared wavelengths. By comparing the positions of the candidate massive YSOs with those of the UCHII regions, we find that 3 massive YSOs in our sample are coincident with a radio source (see Table 6, Col. 5), although the radio counterpart of one of them, $06084 \mathrm{nr} 118$, does not have the radio properties of a typical UCHII region (see Sect. 4.3.3).

To illustrate this, in panel (a) and (b) of Fig. 4 two sources are displayed with a radio counterpart, while panel (c) shows an example of a region where the radio source is not the counterpart of the massive YSO, but of a more embedded source. In panel (a), the $K$-band image of IRAS $06061+2151$ demonstrates that $06061 \mathrm{nr} 676$ is part of a larger cluster and the only source coincident with a radio source (source A: G188.796+01.030). Panel (b) shows the $K$-band image of IRAS 06412-0105 where $06412 \mathrm{nr} 121$ falls in the center of the radio emission. Because of the large distance to this source $(7.3 \mathrm{kpc})$, the extent of the region $(0.8 \mathrm{pc})$ suggests that this region is not an UCHII but a more evolved $\mathrm{H}$ II region.

\section{Discussion}

We have observed a large sample of candidate massive YSOs located in high-mass star-forming regions. The observed photometric and spectral characteristics show that these objects are surrounded by circumstellar material. The next question to address is whether this material is located in a circumstellar disk and, if this is the case, whether these disks are remnants of the accretion process, or formed as a consequence of the mass-loss process and rapid rotation such as in $\mathrm{Be}$ and $\mathrm{B}[\mathrm{e}]$ stars.

\subsection{Origin of the spectral lines}

The different spectral lines observed in the $K$-band spectra of the candidate massive YSOs are formed in regions with different 
physical conditions. These conditions provide information on the origin of the material. In some of the objects the spectral lines indicate that they are formed in a rotating disk (see Table 6, Col. 6).

\subsubsection{High-density indicators}

The special conditions required to emit the $\mathrm{CO}$ first-overtone emission are suggestive of an origin in a circumstellar disk. The $\mathrm{CO}$ is only emitted at high density because the critical density of the $\mathrm{CO}$ overtone transitions is high $\left(10^{10}-10^{11} \mathrm{~cm}^{-3}\right)$. The excitation temperature has to be high as well (2000-4000 K). The disk hypothesis is supported by the shape of the CO bandheads of two objects (16164nr3636 and 18006nr766). They show a CO bandhead profile displaying a blue wing, which can be explained by CO gas in Keplerian rotation (Bik \& Thi 2004).

$\mathrm{CO}$ bandheads can also be produced in a shock front. This is suggested for the BN object where Scoville et al. (1983) propose that the $\mathrm{CO}$ is formed in the shock-front between the $\mathrm{H}$ II region and the ambient molecular cloud. Another tracer of shocks is $\mathrm{H}_{2}$, which is also observed towards our candidate massive YSOs. However, the $\mathrm{H}_{2}$ is spatially extended, and possibly formed in the photo-dissociation region by UV excitation. The $\mathrm{CO}$ is only observed towards the point sources, suggesting that the $\mathrm{CO}$ and $\mathrm{H}_{2}$ emission are not formed by the same process.

Pfund-lines probe high-density material which is also an indication for a disk-like geometry. This is confirmed for the Pflines in $16571 \mathrm{nr} 1281$, which are double peaked, indicating that the lines are emitted in a disk. The Pf-lines in 16164nr3636 and 18006 nr766 have a width comparable to that of the CO bandheads. This suggests that the lines share the same kinematics. They cannot be formed in the same region, as the Pf-lines are formed in ionised gas with a temperature too high to coexist with $\mathrm{CO}$ molecules. This leads to the suggestion that, in case of a circumstellar disk, the $\mathrm{CO}$ is formed in the neutral mid-plane, and the Pf-lines originate in the ionised upper layers.

Two of our reference objects, S106 and NGC 2024-IRS2, show similar bandhead profiles. In these objects also other evidence for a circumstellar disk is found. In the case of S106, evidence for a disk comes from the observed geometry of the extended optical and near-infrared emission (cf. Smith et al. 2001). For NGC 2024-IRS2, the infrared SED points to a (gaseous) disk (Lenorzer et al. 2004).

\subsubsection{Origin of $\operatorname{Br} \gamma$ emission}

The majority of the objects, however, do not show Pfund or CO emission and therefore might have another geometry of the circumstellar material. The $\mathrm{Br} \gamma$ line, however, which is present in all the spectra, shows a remarkably similar behaviour in all objects. This might suggest that the formation mechanism of the $\mathrm{Br} \gamma$ line could be similar for all objects. Especially the $F W H M$ of $\mathrm{Br} \gamma$ is rather constant, it varies between 100 and $220 \mathrm{~km} \mathrm{~s}^{-1}$. The EW of $\mathrm{Br} \gamma$ ranges from 1 to $10 \AA$, except in 3 objects where the EW is much larger (upto $100 \AA$, see Table 3 ). Similar EW values are found in Be stars, where the larger EWs are found for the objects with the earlier spectral type (B0-B2) (Clark \& Steele 2000).

If the circumstellar matter is, like the $\mathrm{CO}$ and Pf-lines suggest, in the shape of a circumstellar disk, Bry emission may be produced in the ionised upper layers of the disk. The Br $\gamma$ emission will be formed over a fairly large area of the circumstellar disk and the emission will be dominated by the largest surface area. This in contrast to the Pf-lines which are only formed in the high-density inner parts of a disk, which makes them much broader than the $\mathrm{Br} \gamma$ line (Table 4).

The width of the $\mathrm{Br} \gamma$ line suggests that the line is not formed in the H II region itself. The sound speed in (ultra) compact H II regions is too low to account for the $\operatorname{Br} \gamma$ line widths. For an isothermal $\mathrm{HII}$ region of $10000 \mathrm{~K}$, the sound speed is about $17 \mathrm{~km} \mathrm{~s}^{-1}$. We note that in hyper-compact $\mathrm{H}$ II regions broad radio recombination lines up to $180 \mathrm{~km} \mathrm{~s}^{-1}$ are observed (Kurtz \& Franco 2002), which probably reflects their expansion velocity. These objects are heavily embedded, much more than the UCHII regions and will, very likely, not be visible in the near-infrared.

The large width of the Bry line could also be explained by a bipolar outflow. The red-shifted and the blue-shifted lobes tilted in the line of sight would then be responsible for the observed width. However, the other spectral lines which then also should be produced in these outflows have different $F W H M$ s, some are broader and some are even unresolved. This would be unlikely in the case of a bipolar outflow. Additionally, our objects show a very strong continuum which is not observed in that case.

Also a normal stellar wind as observed for OB stars would not explain the $\mathrm{Br} \gamma$ emission lines. Only in supergiants the $\mathrm{Br} \gamma$ line is in emission. But these stellar winds have terminal velocities of the order of $1500 \mathrm{~km} \mathrm{~s}^{-1}$. Supergiants are not expected in young star-forming regions. If the lines are formed in a stellar wind, Br $\gamma$ would be broader than the Pf-lines (Lamers et al. 1996), which is not seen in the data (Table 4).

It is also possible that the $\mathrm{Br} \gamma$ line is formed in a disk-wind (Drew et al. 1998). If the central star is emitting a lot of UV radiation, it will ionise the surface of the disk so that the disk evaporates (see Sect. 5.3). This will then result in a dense and low velocity disk wind, where the outflow velocity will soon dominate the rotational velocity. The line widths predicted by Drew et al. (1998) are of the order of $200 \mathrm{~km} \mathrm{~s}^{-1}$. This scenario would also explain the difference in width of the $\mathrm{CO}$ and $\mathrm{Br} \gamma$ lines in $08576 \mathrm{nr} 292$ and 11097nr1218, where the CO can be formed in a disk seen face on and $\mathrm{Br} \gamma$ is originating from a disk-wind.

Summarising, the broad CO emission as well as the doublepeaked Pf-lines strongly suggest that the material in these objects is located in a circumstellar disk. By comparing the behaviour of Bry in all the objects, the origin in a disk-wind seems the most plausible explanation. This means that all the objects may be surrounded by a circumstellar disk. The absence of $\mathrm{CO}$ bandheads in the spectra of most of the objects might therefore be related to the special conditions required to emit the first-overtone emission, rather than the abundance of the $\mathrm{CO}$ molecules.

\subsection{Nature of the objects}

The aim of this project is to identify the ionising source(s) of UCHII regions, and to determine their physical nature. The regions were selected based on the detection of an UCHII radio source and its connection with an IRAS point source fulfilling the UCHII colour-colour criterion of Wood \& Churchwell (1989a). The near-infrared surveys of UCHII regions performed by Hanson et al. (2002) and Kaper et al. (2006) demonstrate that only half of the UCHII radio sources show a near-infrared counterpart. After comparison of the positions of the candidate massive YSOs with those of the UCHII radio positions, only a few objects turn out to be the actual near-infrared counterpart of an ultra-compact radio source (see Sect. 4.3). All objects, however, are part of more extended sites of (massive) star formation which also host UCHII regions. 
The fact that the candidate massive YSOs are located inside regions of star formation suggests that these objects are young. However, also around main sequence OB stars, disks are observed ( $\mathrm{Be}$ and $\mathrm{B}[\mathrm{e}]$ stars). The densities derived for the disks of Be stars are too low to emit the CO bandheads $\left(\sim 10^{10} \mathrm{~cm}^{-3}\right.$, Waters et al. 1991). Also disk-winds are not observed in Be stars. Studies of Be star abundances in open clusters suggest that the Be phenomenom mainly occurs in the second half of their main sequence lifetime (Fabregat \& Torrejón 2000). In B[e] stars, CO emission is observed; Kraus \& Lamers (2003) show that in a very dense, non-spherical stellar wind, such densities can be reached. Only supergiants have these strong stellar winds. None of the candidate massive YSOs is found near the location of the $\mathrm{B}[\mathrm{e}]$ supergiants in the CMD (Fig. 1).

The arguments presented above suggest that the majority of these objects are young and their circumstellar disks are likely a product of the formation process of these massive stars. These disks might be the remnant of the extended accretion disks detected in earlier phases of massive star formation (e.g. Shepherd et al. 2001; Beltrán et al. 2004; Garay \& Lizano 1999; Chini et al. 2004; Jiang et al. 2005; Patel et al. 2005).

\subsection{Disruption of disks around young massive stars}

In some of the star-forming regions where the massive YSOs are detected, also massive stars are found which already cleared their circumstellar environment. Observations of M 17 (Hanson et al. 1997) as well as giant H II regions (Blum 2003) give similar results.

In our spectroscopic sample we find that most of the O stars "already" cleared out their environment while a relatively larger fraction of the B stars are still surrounded by circumstellar material. This leads to the suggestion that the most massive stars clear out their environment fastest.

This trend is also seen when inspecting the location of the massive YSOs in the CMD. The majority of the massive YSOs have a position in the CMD indicating spectral type B. A few objects, however, show evidence that the central star is a hot $\mathrm{O}$ star (see Sect. 4.3). The location of these sources in the CMD is near S106 for which a spectral type O6-O8 has been proposed (e.g. van den Ancker et al. 2000). Note that the position of NGC 2024IRS2 also suggests an O star as the central source. However, when taking into account a strong $K$-band excess and the identification of the main ionising source of NGC 2024, NGC 2024IRS2b (O8), it is more likely that NGC 2024-IRS2 is an early B star (Bik et al. 2003; Lenorzer et al. 2004).

The objects where the central star likely is an $\mathrm{O}$ star show a $(J-K)$ colour which is on average much bluer than those of the objects which are of spectral type B. This difference in colour suggests that the disk properties of these objects are different. $\mathrm{O}$ stars produce much more EUV radiation and possess stronger stellar winds than B stars, which shortens the destruction timescale of disks around $\mathrm{O}$ stars.

Hollenbach et al. (1994, 2000) investigated the different destruction mechanisms of disks around young stars. For OB stars, the outer regions of the disk will be photoevaporated. The extreme-UV photons ionise the top-layer of the disk. This gas will be heated to $10^{4} \mathrm{~K}$ and obtain a velocity dispersion given by the local sound speed $\left(\sim 10 \mathrm{~km} \mathrm{~s}^{-1}\right)$. Near the star this velocity will be smaller than the escape velocity. The gravitational radius $\left(r_{\mathrm{g}}\right)$, the radius at which the sound-speed is equal to the escape velocity ranges from $\sim 500 \mathrm{AU}$ for $\mathrm{O} 3$ stars to $100 \mathrm{AU}$ for early $\mathrm{B}$ (Table 7). Beyond the gravitational radius, the sound speed
Table 7. Two different effects are responsible for the destruction of circumstellar disks around $\mathrm{O}$ stars; the photo-evaporation process and the direct interaction between the wind and the disk. In the first 3 columns, the stellar parameters are given used in the calculation of the timescales (Mokiem et al. 2004; Lenorzer et al. 2004). In Col. 4, the gravitational radius is given. Beyond this radius matter is removed by photoevaporation, while inside of it, the stellar wind directly removes material from the disk. Cols. 5 and 6 give the typical timescales for these two processes. The formulae are taken from Hollenbach et al. (1994, 2000).

\begin{tabular}{lclccl}
\hline \hline SpTp & $\begin{array}{c}\log \left(Q_{0}\right) \\
\mathrm{s}^{-1}\end{array}$ & $\begin{array}{c}\log (\dot{M}) \\
M_{\odot} \mathrm{yr}^{-1}\end{array}$ & $\begin{array}{c}r_{\mathrm{g}} \\
\mathrm{AU}\end{array}$ & $\begin{array}{c}t_{\mathrm{p}} \\
10^{5} \mathrm{yr}\end{array}$ & $\begin{array}{c}t_{\mathrm{w}} \\
10^{5} \mathrm{yr}\end{array}$ \\
\hline O3V & 49.69 & -5.375 & 459.5 & 0.6 & 0.7 \\
O4V & 49.50 & -5.599 & 377.4 & 0.8 & 1.1 \\
O5V & 49.31 & -5.805 & 301.4 & 0.9 & 1.8 \\
O5.5V & 49.08 & -6.072 & 251.4 & 1.1 & 3.3 \\
O6.5V & 48.82 & -6.369 & 205.4 & 1.3 & 6.5 \\
O7.5V & 48.51 & -6.674 & 169.4 & 1.7 & 13.3 \\
O9V & 48.06 & -7.038 & 141.4 & 2.5 & 30.7 \\
O9.5V & 47.79 & -7.252 & 128.7 & 3.3 & 50.1 \\
O9.7V & 47.52 & -7.445 & 118.0 & 4.2 & 78.8 \\
B1V & 46.92 & -7.913 & 98.7 & 7.3 & 233.5 \\
\hline
\end{tabular}

will exceed the escape velocity so that the matter can freely escape from the disk, e.g. in the form of a disk wind.

This model discriminates between two scenarios, the weak wind case and the strong wind case. For massive stars, the strong-wind case is applicable. In this strong-wind case, the disk is flattened by the stellar wind. The strong stellar wind prohibits that ionised gas located beyond $r_{\mathrm{g}}$ is freely escaping from the disk surface, but is dragged over the surface of the disk to the point where the ram pressure of the wind balances the thermal pressure of the ionised hydrogen. From there on, the gas can freely escape from the disk. This strong-wind case leads to a faster destruction of the disk than the weak-wind case.

The timescale as derived by Hollenbach et al. (1994) depends on the number of ionising continuum photons, the massloss rate, the wind velocity and the mass of the disk. The stellar parameters are taken from Mokiem et al. (2004) and Lenorzer et al. (2004). By assuming a disk mass of 2 solar masses (see Hollenbach et al. 1994; Yorke \& Welz 1996), the destruction timescale ranges from somewhat less than $10^{5}$ year for a disk around an early $\mathrm{O}$ star, to $\sim 5 \times 10^{5}$ year for a disk surrounding a B0 V star (Table 7). The assumption in this model is that the material in the disk is not replenished, and that accretion has stopped. These short timescales indicate that already very soon after the accretion stops, the outer disk regions will be rapidly destroyed by the EUV photons of the star.

For the destruction of the regions located within the gravitational radius, the direct interaction with the stellar wind is much more important. By considering the momentum transferred by the collision of a spherical wind with the disk Hollenbach et al. (2000) derive a timescale for this process. Typical timescales for the destruction of the inner parts of the disk by the interaction with the stellar wind are given in Col. 6 of Table 7. The mass of the disk inside this gravitational radius is taken to be $10 \%$ of the total disk mass.

Comparison of the two timescales (Table 7) shows that first the outer regions of the disk are destroyed by photo-evaporation and that the inner parts of the disk are removed at a later stage. So observationally one should look for indicators of a remnant disk by probing material near the star, i.e. warm gas and dust, and not the cold material probed at mm wavelengths. 
The detected number of massive YSOs which harbour an O star compared to the amount of massive YSOs which are of spectral type $\mathrm{B}$ is qualitatively consistent with the trend suggested by this model. The destruction of the disks around young O stars is much faster than those around B stars, so the probability to detect an $\mathrm{O}$ star surrounded by a remnant accretion disk is much smaller than such a disk around a young B star. This suggests that these objects are really young massive stars surrounded by a remnant accretion disk. Observations of Herbig Be stars also show the effect of the outer parts of the disk being photoevaporated by the UV photons. The disk masses, if detected at all, are substantially lower than those around Herbig Ae stars (Natta et al. 2000; Fuente et al. 2003).

\section{Conclusions}

In this paper, high-resolution $K$-band spectra of candidate massive YSOs located in high-mass star-forming regions are presented. The results can be summarised as follows:

- The near-infrared photometric properties of the massive YSO candidates suggest the presence of a near-infrared excess. This implies that large amounts of circumstellar matter are present around these objects.

- This prediction is confirmed by VLT/ISAAC $K$-band spectroscopy. The objects were selected on the presence of $\mathrm{Br} \gamma$ emission, but also show other emission lines formed in a warm circumstellar environment like Fe II, Mg II, Pf 23-5 Pf 33-5 and CO first-overtone bandhead emission. In some objects also He I emission is detected.

- In general these candidate massive YSOs are not the ionising sources of UCHII radio sources. Only 3 sources are associated with an UCHII radio source. All the objects, however, are located in more extended $\mathrm{H}$ II regions; in most cases also OB stars are found which already cleared out their environment. The UCHII radio sources located in these fields are not visible in the near-infrared, likely because they are deeply embedded.

- The location of these objects inside high-mass star-forming regions, with associated UCHII radio sources, suggests that these objects are still in an early evolutionary phase, although the objects may be more evolved than the ionising sources of the UCHII regions which often remain undetected. The massive YSOs found in M 17 and a sample of giant $\mathrm{H}$ II regions suggest that these objects have an age of about $10^{6}$ year.

- Most of the stars have near-infrared colours similar to the Herbig Be stars, suggesting that also most of the stars in our sample are of spectral type B. For 5 objects, however, the location in the CMD (Fig. 1) suggests that the central stars are of spectral type O. For two sources this is supported by circumstantial evidence provided by the nebular emission lines and IRAS infrared flux.

- Photo-evaporation and disk destruction models presented by Hollenbach et al. $(1994,2000)$ predict that O stars clear their circumstellar environment very soon after the accretion has stopped. For B stars the timescales are substantially longer. This is consistent with the detection of only a few massive YSOs with spectral type O.

- The Br $\gamma$ line likely originates in an ionised disk wind, while the Pf lines are probably emitted in the inner regions of a disk. The $\mathrm{CO}$ emission lines are only produced in a very dense medium, and are likely forming in the dense mid plane of the disk.
- The high density to emit the $\mathrm{CO}$ bandheads as well as the origin of $\mathrm{Br} \gamma$ in a disk-wind do not point to an origin in a massloss disk such as present around Be stars. These conditions are met in the dense non-spherical winds of $\mathrm{B}[\mathrm{e}]$ supergiants, but the location of the sources in high-mass star-forming regions as well as their location in the CMD (Fig. 1) are not consistent with a possible supergiant nature.

- The evidence presented in this paper suggests that the infrared excess is created by a remnant accretion disk, rather than a mass-loss disk. These disks are likely the remnants of the large accretion disks found around high mass stars in hot cores (Minier et al. 1998; Shepherd et al. 2001; Beltrán et al. 2004; Chini et al. 2004).

Acknowledgements. A.B. acknowledges financial support from the DFG during a two-month visit at ESO Headquaters. L.K. acknowledges the support of a fellowship of the Royal Academy of Arts and Sciences in The Netherlands. The authors thank the VLT staff for support and help with the observations, Margaret Hanson for help with the observations and datareduction process, Annique Lenorzer, Wing-Fai Thi, Stan Kurtz and Fernando Comerón for helpful discussions. Andrew Walsh and Ed Churchwell are thanked for providing the radio images. We thank the referee for his/her suggestions to improve the paper. NSO/Kitt Peak FTS data used here were produced by NSF/NOAO.

\section{References}

Bally, J., Snell, R. L., \& Predmore, R. 1983, ApJ, 272, 154

Barbosa, C. L., Damineli, A., Blum, R. D., \& Conti, P. S. 2003, AJ, 126, 2411

Beltrán, M. T., Cesaroni, R., Neri, R., et al. 2004, ApJ, 601, L187

Bik, A., \& Thi, W. F. 2004, A\&A, 427, L13

Bik, A., Lenorzer, A., Kaper, L., et al. 2003, A\&A, 404, 249

Bik, A., Kaper, L., Hanson, M. M., \& Smits, M. 2005, A\&A, 440, 121

Blum, R. D. 2003, in A massive star odyssey: from main sequence to supernova, IAU Symp., 212, 458

Blum, R. D., Damineli, A., \& Conti, P. S. 1999, AJ, 117, 1392

Blum, R. D., Conti, P. S., \& Damineli, A. 2000, AJ, 119, 1860

Blum, R. D., Damineli, A., \& Conti, P. S. 2001, AJ, 121, 3149

Blum, R. D., Barbosa, C. L., Damineli, A., Conti, P. S., \& Ridgway, S. 2004, ApJ, 617, 1167

Bonnell, I. A., Bate, M. R., \& Zinnecker, H. 1998, MNRAS, 298, 93

Bowen, I. S. 1947, PASP, 59, 196

Brand, J., \& Blitz, L. 1993, A\&A, 275, 67

Bronfman, L., Nyman, L.-A., \& May, J. 1996, A\&AS, 115, 81

Carpenter, J. M., Snell, R. L., \& Schloerb, F. P. 1995, ApJ, 450, 201

Carr, J. S. 1989, ApJ, 345, 522

Caswell, J. L., \& Haynes, R. F. 1987, A\&A, 171, 261

Chandler, C. J., Carlstrom, J. E., Scoville, N. Z., Dent, W. R. F., \& Geballe, T. R. 1993, ApJ, 412, L71

Chandler, C. J., Carlstrom, J. E., \& Scoville, N. Z. 1995, ApJ, 446, 793

Chini, R., Hoffmeister, V., Kimeswenger, S., et al. 2004, Nature, 429, 155

Chini, R., \& Neckel, T. 1981, A\&A, 102, 171

Churchwell, E. 1991, in The Physics of Star Formation and Early Stellar Evolution, NATO ASIC Proc., 342: 221

Churchwell, E. 2002, ARA\&A, 40, 27

Clark, J. S., \& Steele, I. A. 2000, A\&AS, 141, 65

Conti, P. S., \& Blum, R. D. 2002, ApJ, 564, 827

Cox, A. N. 2000, Allen's astrophysical quantities, Allen's astrophysical quantities, 4th ed. (New York: AIP Press; Springer), ed. Arthur N. Cox

Dougherty, S. M., Waters, L. B. F. M., Burki, G., et al. 1994, A\&A, 290, 609

Drew, J. E., Proga, D., \& Stone, J. M. 1998, MNRAS, 296, L6

Eiroa, C., Oudmaijer, R. D., Davies, J. K., et al. 2002, A\&A, 384, 1038

Eisner, J. A., Lane, B. F., Akeson, R. L., Hillenbrand, L. A., \& Sargent, A. I. 2003, ApJ, 588, 360

Fabregat, J. \& Torrejón, J. M. 2000, A\&A, 357, 451

Felli, M., Massi, M., Staude, H. J., et al. 1984, A\&A, 135, 261

Figuerêdo, E., Blum, R. D., Damineli, A., \& Conti, P. S. 2002, AJ, 124, 2739

Figuerêdo, E., Blum, R. D., Damineli, A., \& Conti, P. S. 2005, AJ, 129, 1523

Fuente, A., Rodríguez-Franco, A., Testi, L., et al. 2003, ApJ, 598, L39

Gómez, Y., Rodríguez, L. F., \& Garay, G. 2002, ApJ, 571, 901

Garay, G., \& Lizano, S. 1999, PASP, 111, 1049

Geballe, T. R., \& Persson, S. E. 1987, ApJ, 312, 297

Grasdalen, G. L. 1974, ApJ, 193, 373

Hanson, M. M. 1998, in Properties of Hot Luminous Stars, ASP Conf. Ser., 131, 
Hanson, M. M., Conti, P. S., \& Rieke, M. J. 1996, ApJS, 107, 281

Hanson, M. M., Howarth, I. D., \& Conti, P. S. 1997, ApJ, 489, 698

Hanson, M. M., Luhman, K. L., \& Rieke, G. H. 2002, ApJS, 138, 35

Hollenbach, D., Johnstone, D., Lizano, S., \& Shu, F. 1994, ApJ, 428, 654

Hollenbach, D. J., Yorke, H. W., \& Johnstone, D. 2000, Protostars and Planets IV, 401

Hony, S., Waters, L. B. F. M., Zaal, P. A., et al. 2000, A\&A, 355, 187

Jiang, Z., Tamura, M., Fukagawa, M., et al. 2005, Nature, 437, 112

Kaper, L., Bik, A., Comerón, F., \& Hanson, M. M. 2006, A\&A, submitted

Karnik, A. D., Ghosh, S. K., Rengarajan, T. N., \& Verma, R. P. 2001, MNRAS, 326, 293

Koempe, C., Baudry, A., Joncas, G., \& Wouterloot, J. G. A. 1989, A\&A, 221, 295

Kraus, M., \& Lamers, H. J. G. L. M. 2003, A\&A, 405, 165

Kurtz, S., \& Franco, J. 2002, in Rev. Mex. Astron. Astrof. Conf. Ser., 16

Kurtz, S., Churchwell, E., \& Wood, D. O. S. 1994, ApJS, 91, 659

Lamers, H. J. G. L. M., Najarro, F., Kudritzki, R. P., et al. 1996, A\&A, 315, L229

Lamers, H. J. G. L. M., Zickgraf, F., de Winter, D., Houziaux, L., \& Zorec, J. 1998, A\&A, 340, 117

Lenorzer, A., Bik, A., de Koter, A., et al. 2004, A\&A, 414, 245

Liseau, R., Lorenzetti, D., Nisini, B., Spinoglio, L., \& Moneti, A. 1992, A\&A, 265,577

Mannings, V., \& Sargent, A. I. 1997, ApJ, 490, 792

Martín-Hernández, N. L., Bik, A., Kaper, L., Tielens, A. G. G. M., \& Hanson, M. M. 2003, A\&A, 405, 175

McGregor, P. J., Hyland, A. R., \& Hillier, D. J. 1988, ApJ, 324, 1071

Millan-Gabet, R., Schloerb, F. P., \& Traub, W. A. 2001, ApJ, 546, 358

Minier, V., Booth, R. S., \& Conway, J. E. 1998, A\&A, 336, L5

Mokiem, M. R., Martín-Hernández, N. L., Lenorzer, A., de Koter, A., \& Tielens, A. G. G. M. 2004, A\&A, 419, 319

Morris, P. W., Eenens, P. R. J., Hanson, M. M., Conti, P. S., \& Blum, R. D. 1996, ApJ, 470, 597

Natta, A., Grinin, V., \& Mannings, V. 2000, Protostars and Planets IV, 559

Neckel, T., \& Staude, H. J. 1984, A\&A, 131, 200

Patel, N. A., Curiel, S., Sridharan, T. K., et al. 2005, Nature, 437, 109

Pelupessy, I., Lamers, H. J. G. L. M., \& Vink, J. S. 2000, A\&A, 359, 695
Persi, P., \& Tapia, M. 2003, A\&A, 406, 149

Rousselot, P., Lidman, C., Cuby, J.-G., Moreels, G., \& Monnet, G. 2000, A\&A, 354,1134

Scoville, N. Z., Hall, D. N. B., Ridgway, S. T., \& Kleinmann, S. G. 1979, ApJ, 232, L121

Scoville, N., Kleinmann, S. G., Hall, D. N. B., \& Ridgway, S. T. 1983, ApJ, 275, 201

Shepherd, D. S., Claussen, M. J., \& Kurtz, S. E. 2001, Science, 292, 1513

Shu, F. H., Adams, F. C., \& Lizano, S. 1987, ARA\&A, 25, 23

Simpson, J. P., \& Rubin, R. H. 1990, ApJ, 354, 165

Smith, N., Jones, T. J., Gehrz, R. D., Klebe, D., \& Creech-Eakman, M. J. 2001, AJ, 121, 984

Stee, P., Vakili, F., Bonneau, D., \& Mourard, D. 1998, A\&A, 332, 268

Storey, P. J., \& Hummer, D. G. 1995, MNRAS, 272, 41

Tan, J. C. 2004, ApJ, 607, L47

Tapia, M. 1991, Mem. Soc. Astron. Ital., 62, 731

Vakili, F., Mourard, D., Stee, P., et al. 1998, A\&A, 335, 261

van der Ancker, M. E. 2004, in High Resolution Infrared Spectroscopy in Astronomy, ed. H. U. Käufl, R. Siebenmorgen, \& A. Moorwood, 309

van den Ancker, M. E., Tielens, A. G. G. M., \& Wesselius, P. R. 2000, A\&A, 358,1035

Vandenbussche, B., Beintema, D., de Graauw, T., et al. 2002, A\&A, 390, 1033

Voors, R. H. M. 1999, Ph.D. Thesis, University of Amsterdam

Walsh, A. J., Burton, M. G., Hyland, A. R., \& Robinson, G. 1998, MNRAS, 301, 640

Walsh, A. J., Hyland, A. R., Robinson, G., \& Burton, M. G. 1997, MNRAS, 291, 261

Waters, L. B. F., Marlborough, J. M., van der Veen, W. E. C., Taylor, A. R., \& Dougherty, S. M. 1991, A\&A, 244, 120

Wolfire, M. G., \& Cassinelli, J. P. 1987, ApJ, 319, 850

Wood, D. O. S., \& Churchwell, E. 1989a, ApJ, 340, 265

Wood, D. O. S., \& Churchwell, E. 1989b, ApJS, 69, 831

Yorke, H. W., \& Sonnhalter, C. 2002, ApJ, 569, 846

Yorke, H. W., \& Welz, A. 1996, A\&A, 315, 555

Zickgraf, F.-J., Wolf, B., Stahl, O., Leitherer, C., \& Klare, G. 1985, A\&A, 143, 421 